\title{
A Review for Compact Model of Thin-Film Transistors (TFTs)
}

\author{
Nianduan Lu ${ }^{1,2,3}$, Wenfeng Jiang ${ }^{1,2,3}$, Quantan Wu $\mathrm{Wu}^{1,2,3}$, Di Geng ${ }^{1,2,3}$, Ling $\mathrm{Li}^{1,2,3, *}$ and \\ Ming Liu ${ }^{1,2,3}$ \\ 1 Key Laboratory of Microelectronic Devices \& Integrated Technology, Institute of Microelectronics, \\ Chinese Academy of Sciences, Beijing 100029, China; lunianduan@ime.ac.cn (N.L.); \\ jiangwenfeng1912@163.com (W.J.); wuquantan@ime.ac.cn (Q.W.); gengdi@ime.ac.cn (D.G.); \\ liuming@ime.ac.cn (M.L.) \\ 2 School of Microelectronics, University of Chinese Academy of Sciences, Beijing 100049, China \\ 3 Jiangsu National Synergetic Innovation Center for Advanced Materials (SICAM), Nanjing 210009, China \\ * Correspondence: lingli@ime.ac.cn
}

Received: 16 October 2018; Accepted: 9 November 2018; Published: 15 November 2018

\begin{abstract}
Thin-film transistors (TFTs) have grown into a huge industry due to their broad applications in display, radio-frequency identification tags (RFID), logical calculation, etc. In order to bridge the gap between the fabrication process and the circuit design, compact model plays an indispensable role in the development and application of TFTs. The purpose of this review is to provide a theoretical description of compact models of TFTs with different active layers, such as polysilicon, amorphous silicon, organic and In-Ga-Zn-O (IGZO) semiconductors. Special attention is paid to the surface-potential-based compact models of silicon-based TFTs. With the understanding of both the charge transport characteristics and the requirement of TFTs in organic and IGZO TFTs, we have proposed the surface-potential-based compact models and the parameter extraction techniques. The proposed models can provide accurate circuit-level performance prediction and RFID circuit design, and pass the Gummel symmetry test (GST). Finally; the outlook on the compact models of TFTs is briefly discussed.
\end{abstract}

Keywords: thin-film transistors (TFTs); compact model; surface potential

\section{Introduction}

A thin-film transistors (TFTs) is a special kind of field-effect transistor (FET) fabricated by depositing thin films of an active semiconductor layer, as well as the dielectric layer and metallic contacts over a supporting (but non-conducting) substrate [1,2]. In the past 15 years, TFTs has grown into a huge industry based on display, memory, E-paper applications, and so on [3-6]. Generally, a common substrate in TFTs is glass, which differs from the conventional transistor, where the semiconductor material typically is the substrate, such as a silicon wafer. TFTs include three basic elements: (1) a thin semiconductor film; (2) an insulating layer; and (3) three electrodes (gate, source and drain) [7-9]. Three basic elements for configuration of TFT have been illustrated clearly in Figure 1. The source and drain, are in contact with the semiconductor film at a short distance from one another. The gate is separated from the semiconductor film by the insulating layer [10]. 


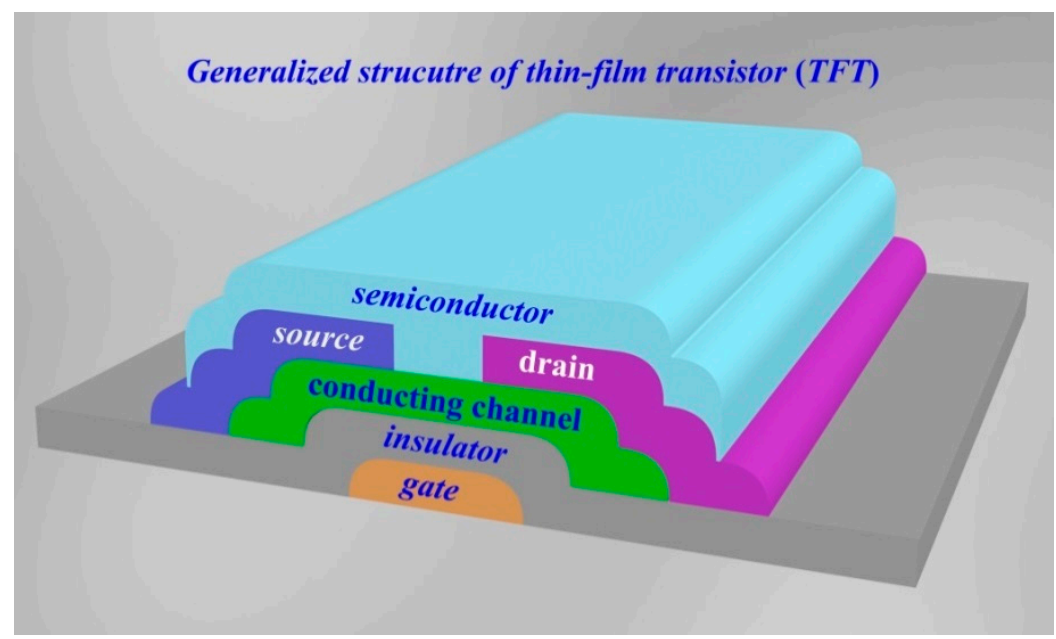

Figure 1. Schematic structure of a generalized thin-film transistor.

The history of TFT really began with the work of P. K. Weimer at Radio Corporation of America (RCA) Laboratories in 1962 [11]. At that time Weimer fabricated the first TFT based on thin films of polycrystalline cadmium sulfide as the semiconductor materials. In the 1970s, the realization of crystalline silicon as the active materials with low cost dramatically changed the prospects of TFTs [12]. In 1979, amorphous silicon as a new active material was introduced by LeComber et al. [13], which had profound implications for TFTs. In 1980, Depp et al. reported polysilicon TFT which achieved good mobility and TFT characteristics [14]. In 1986, the first transistor based on organic semiconductor was reported [15]. As compared with conventional Si TFTs, organic TFT (OTFT) displays much less complex in fabrication processes and can be naturally compatible with plastic substrates for lightweight and foldable products [16]. To develop large-scale TFTs, processing temperatures must be getting lower and lower. In 2004, Nomura et al. used a complex In-Ga-Zn-O (IGZO) semiconductor layer in a TFT, which achieved the room-temperature processing of the semiconductor layer [17]. Looking back into the past half-century, TFTs moved endlessly forward from the initial requirement of performance to today's application of large area and low cost.

During the development of TFTs, the semiconductor device model represents an essential bridge between the semiconductor manufactures and the circuit design. Integrated circuit (IC) designers usually utilize various kinds of software (such as Cadence, SPICE, PHILIPAC) for design circuit [18-20]. The core of the corresponding software is the model of each unit device. Because the IC is consisted of several transistors, if all unit devices would need to run the complicated model of transistor, the system level simulation will beyond computer ability and hence causes non-convergence in calculation. Otherwise, for ensuring the reliability of the simulation, the device model should also be able to accurately describe the physical properties [21]. Compact model is a critical step in the design cycle of modern IC products [22]. It refers to the development of models for integrated semiconductor devices for use in circuit simulations. Compact model is usually used to reproduce device terminal behaviors with accuracy, computational efficiency, ease of parameter extraction, and relative model simplicity for a circuit or system-level simulation, for future technology nodes [23].

Accurate and physical compact models are essential for digital and analog circuits. Generally speaking, an excellent compact model should include the following requirements [21,24]:(i) Representing consistently the behavior; (ii) Being symmetrical to reflect the symmetry of TFT structure; (iii) Being analytical, without differentials or integrals; (iv) Being simple and easily derivable; (v) Parameters that can be characterized easily, or even guessed; (vi) Being upgradable and reducible; (vii) Relations can be physically justified; (viii) Being similar form and correspondence to compact models for other TFTs; (ix) Being tunable to inaccurate (or uncertain) experimental data.

The first compact model could date back to 1983, in which Kacprzak et al. proposed a compact DC model of GaAs FETs for large-signal computer calculation [25]. In 1986, based on one-dimensional (1-D) 
solution of Poisson's equation, Ahmed et al. reported a compact model for accumulation mode poly-Si devices [26]. Later, plenty of methods, such charge sheet model, effective medium approach (EMA), semi-empirical approach, generation-recombination model, and surface-potential based model, have been introduced for the compact models of the silicon-based TFTs [27-32]. Then, with the emergence of new TFTs, e.g., OTFT and IGZO TFTs, some excellent compact models based on interesting methods have been developed [33-37]. Strictly speaking, all of the proposed compact models can be divided into two categories. One is charge-based and another is surface-potential-based. As compared with the charge-based model, the surface-potential-based compact model is believed to have high accuracy and strong physical property, and be easily simplified into the charge-based and threshold-voltage-based model [21]. It can also describe the operation of transistor more accurately without any smooth functions [38].

Over the past two decades, although some excellent reviews have been published [39-42], a completed review for the compact models of TFTs based on different active materials is still lacking. In this review, we will provide an updated review of surface-potential-based compact model of TFTs with different active materials, such as polysilicon, amorphous silicon, organic and IGZO semiconductors. In Section 2, the charge transport property of different active materials is discussed. In Section 3, we discuss the surface-potential-based compact models for silicon-based TFTs and presented our surface-potential-based compact models for organic and IGZO TFTs, respectively. In Section 4, the comparison of various compact models will be summarized. Finally, the future outlook for this field is briefly discussed in Section 5 .

\section{Charge Transport Property}

In order to achieve an accurate compact model for TFTs, the key is to correctly describe the charge transport characteristics. For TFTs with different active materials, the charge transport has displayed various properties. This section will, in detail, introduce the charge transport properties of TFTs for different active materials.

\subsection{Grain-Boundary Trapping Theory}

Based on its structure characteristics, the charge transport property of polysilicon has been described in terms of two distinct models: segregation theory and grain-boundary trapping theory [43]. In the segregation theory, impurity atoms tend to segregate at the grain boundary where they are electrically inactive. While the grain boundary trapping theory assumed that the presence of a large amount of trapping states at the grain boundary able to capture, and therefore immobilize, free carriers. The basic limitation of segregation theory is that it does not explain the temperature dependence of the film resistivity which is thermally activated and exhibits a negative temperature coefficient. The grain-boundary trapping theory can explain most of electrical properties in polysilicon.

In the grain-boundary trapping theory, a polysilicon is assumed to be composed of small crystallites joined together by the grain boundaries usually consisted of a few atomic layers of disordered atoms [43]. Inside each crystallite the atoms are arranged in a periodic manner so that it can be considered as a small single crystal. Atoms in the grain boundary represent a transitional region between the different orientations of neighboring crystallites. Although polysilicon is a three-dimensional substance, it is sufficient to treat the problem in one dimension to calculate the transport properties. The traps are assumed to be initially neutral and become charged by trapping a carrier. Figure 2 shows the schematic diagram of crystal structure, charge distribution and energy band structure of polysilicon films. 

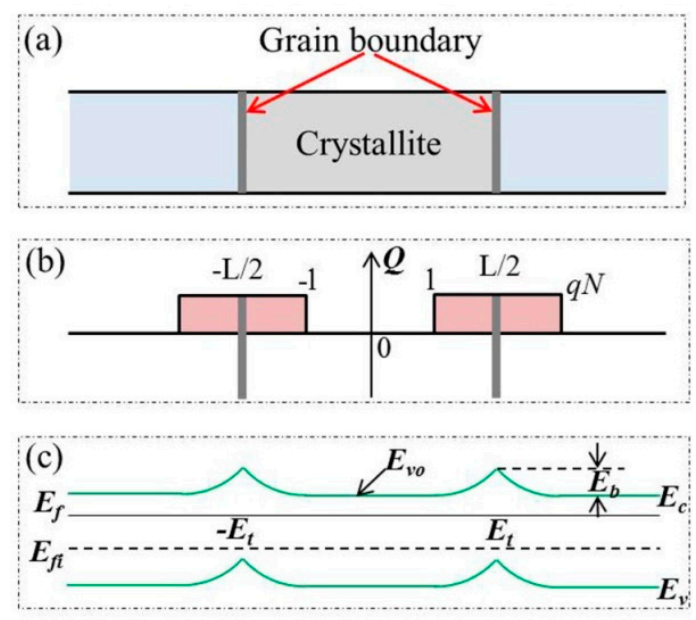

Figure 2. Schematic diagram of (a) crystal structure; (b) charge distribution; and (c) energy band structure of polysilicon films.

The grain-boundary trapping theory considers just the resistance of the grain-boundary region, which includes two important contributions to the current: thermionic emission and tunneling (field emission) [44]. Thermionic emission results from those carriers possessing high enough energy to surmount the potential barrier at the grain boundary. The tunneling current arises from carriers with energy less than the barrier height. When the barrier is narrow and high, the tunneling current can become comparable to or larger than the thermionic emission current. In the polysilicon the potential barrier is the highest when the barrier width is the widest. Because of this, tunneling current may be neglected. Then, for an applied voltage the thermionic emission current density across a grain boundary following Bethe is expressed as the following [44]:

$$
J_{t h}=q p_{a}\left(\frac{k_{B} T}{2 m^{*} \pi}\right)^{1 / 2} \exp \left(-\frac{q V_{B}}{k_{B} T}\right)\left[\exp \left(-\frac{q V_{a}}{k_{B} T}\right)-1\right]
$$

where $q$ is the elemental charge, $p_{a}$ is the average carrier concentration, $m^{*}$ is the effective mass of the carrier, $k_{B}$ is the Boltzmann constant, $V_{B}$ is the potential barrier height, and $V_{a}$ is the applied voltage. Equation (1) neglects collisions within the depletion region and the carrier concentration in the crystallite was assumed to be independent of the current flow, so that it is applicable only if the number of carriers taking part in the current transport is small compared to the total number of carriers in the crystallite. This condition restricts the barrier height to be larger than or comparable to $k_{B} T$. If $V_{a}$ is small, $q V_{a} \ll k_{B} T$, Equation (1) can be expanded to give the following:

$$
J_{t h}=q^{2} p_{a}\left(\frac{1}{2 \pi m^{*} k_{B} T}\right)^{1 / 2} \exp \left(-\frac{q V_{B}}{k_{B} T}\right) V_{a}
$$

which is a linear current-voltage relationship. Based on Equation (2), the conductivity of a polysilicon film with a grain size $L$ is written as:

$$
\sigma=L q^{2} p_{a}\left(\frac{1}{2 \pi m^{*} k_{B} T}\right)^{1 / 2} \exp \left(-\frac{q V_{B}}{k_{B} T}\right)
$$

Then, the effective mobility is expressed as:

$$
\mu_{e f f}=L q\left(\frac{1}{2 \pi m^{*} k_{B} T}\right)^{1 / 2} \exp \left(-\frac{E_{b}}{k_{B} T}\right)
$$

here $E_{b}$ is the energy barrier. 


\subsection{Hopping Transport}

Differing from crystalline materials, such as polysilicon, the charge transport in amorphous materials exhibits very different properties. Amorphous semiconductor materials, including inorganic and organic, have in common, that their atomic or molecular structure is completely disordered. For inorganic amorphous semiconductors, such as, pure and hydrogenated amorphous silicon (a-Si, a-Si:H), a band structure similar to the one of crystalline materials still exists [45,46]. The electronic states in the conduction and valence bands are therefore delocalized. Thus some of the concepts from crystalline semiconductor physics are still suitable for the inorganic amorphous materials. However, in the band gap between valence and conduction band, some localized states exist in which charge carriers can be trapped. For organic amorphous semiconductors, the intermolecular bonds are due to relatively weak van der Waals interactions, the electronic wave functions usually do not extend over the entire volume of the organic solid, but rather, are localized to a finite number of molecules, or even to individual molecules $[47,48]$. Due to the spatial and energetic disorder, the charge transport in amorphous semiconductor materials is limited by trapping in the localized states. This means that the charge carrier mobility is expected to be thermally activated, that is, the charge transport always happens to jump from one localized site to another. This type of transport mechanism is called hopping transport. The transition of hopping between two sites depends on the overlap of the electronic wave functions of these two sites [49]. Whenever a charge carrier hops to a site with a higher (lower) site energy than the site that it came from, the difference in energy is accommodated for by the absorption (emission) of a phonon. Figure 3 is a schematic diagram of carrier hopping transport with the density of states [50].

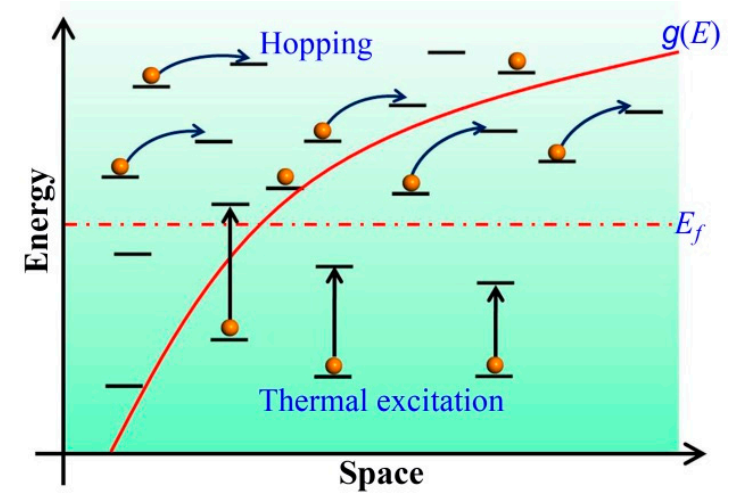

Figure 3. Schematic diagram of hopping transport with the density of states.

The intrinsic transition rate for a carrier hopping from an initial site $i$ to an empty site $j$ is expressed by $\gamma_{i j}=\gamma\left(R_{i j}, E_{i}-E_{j}\right)$. The average transition rate from site $i$ to site $j$ is then [51]:

$$
v_{i j}=<m_{i}\left(1-m_{j}\right) \gamma_{i j}>
$$

where $m_{i}$ and $m_{j}$ are the occupation numbers for sites $i$ and $j$, respectively. The energy dependence of $\gamma_{i j}$ is then a good approximation to take the Miller-Abrahams form to write as [52]:

$$
\gamma_{i j}=v_{0} \exp \left(-2 \varphi R_{i j}-\frac{\theta\left(E_{j}-E_{i}\right)}{k_{B} T}\right)
$$

where $v_{0}$ is the attempt-to-jump frequency, $\varphi$ is the inverse localized length of the inverse wave function, $R_{i j}$ is the distance between site $i$ and site $j, E_{i}$ and $E_{j}$ are the energies of sites $i$ and $j$, respectively, and $\theta(x)=x \varepsilon(x)$ with $\varepsilon(x)$ being the step function. 


\subsection{Multiple Trapping and Release Theory}

For some special materials, such as the small-molecule organic semiconductor and the IGZO semiconductor, which have a strong tendency to form polycrystalline films [50,53,54]. These semiconductors display the regular arrangement, and the delocalized orbitals partially overlap, thereby facilitating more efficient charge transfer and carrier mobility that is much larger than in amorphous films. The charge transport properties of these materials cannot be explained by the grain-boundary trapping theory and hopping transport. In contrast to the grain-boundary trapping theory or hopping theory, the multiple trapping and release (MTR) theory is adapted for the materials $[55,56]$. MTR theory assumes that the charge transport occurs in extended states, and that most of the charge carriers are trapped in localized states [57]. Energy of localized state is separated from mobility edge energy. When the energy of localized state is slightly lower mobility edge, then the extended states acts as shallow trap, from which the charge carrier can be released (emitted) by the thermal excitations. But, if that energy is far below mobility edge energy, then charge carriers cannot be thermally excited (emitted). The number of carriers available for transport depends on the difference in energy between the trap level and the extended-state band. Figure 4 is a transport diagram of MTR theory.

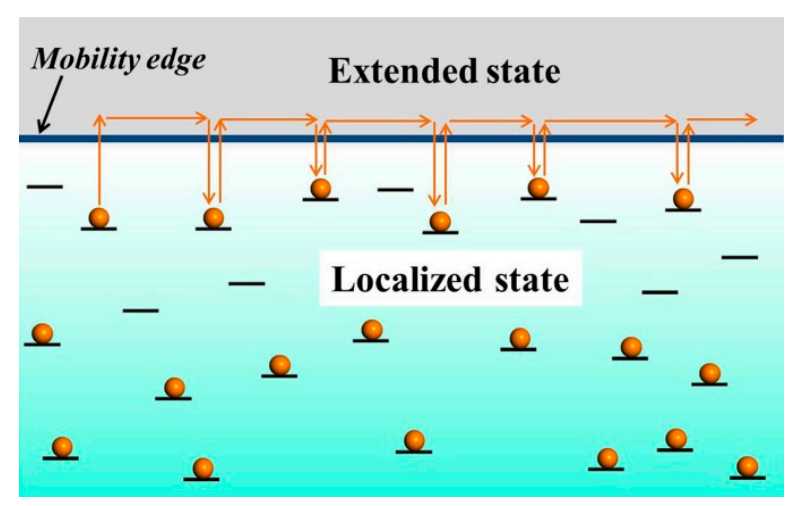

Figure 4. Transport diagram of multiple trapping and release (MTR) theory. The charge carrier (orange balls) is trapped and released into and from localized states (black lines). Conduction happens above the mobility-edge (gray area).

In the MTR theory, total charge carriers' densities, $n_{\text {total }}$, is equal to sum of density in extended states, $n_{e}$, and in localized states, as in Ref. [57]:

$$
n_{\text {total }}=n_{e}+\int_{-\infty}^{0} g(E) f(E) d E
$$

where the upper limit of integral $E=0$ corresponds to the mobility edge, $g(E)$ is the trap density of states (DOS) energy distribution. $f(E)=\left(1+\exp \left(\frac{E-E_{f}(x)}{k_{B} T}\right)\right)^{-1}$ is the Fermi-Dirac distribution, $E_{f}(x)$ is the quasi-Fermi level. Two methods within the MTR theory usually describe the effect of trapping [58]. One is that, all carrier fields induced can contribute to the current flow at any moment of time, but the effective mobility is reduced in comparison with its intrinsic, trap-free value:

$$
\mu_{e f f}=\mu_{0}(T) \frac{\tau(T)}{\tau(T)+\tau_{t r}(T)}
$$

here, $\mu_{0}$ is the carrier mobility in extended state, $\tau_{t r}(T)$ is the average trapping time on shallow traps, and $\tau(T)$ is the average time that a polaron spends diffusively traveling between the consecutive 
trapping events. Another is that only a fraction of the carrier field induced is moving at any given moment of time:

$$
n_{e f f}=n_{t o t a l} \frac{\tau(T)}{\tau(T)+\tau_{t r}(T)}
$$

\section{Surface-Potential-Based Compact Models}

In spite of the fact that the transport characteristics in TFTs is very different for different active materials, the current-voltage characteristics can, to first order, be described with the same formalism as [53]:

$$
I_{d s}=\left\{\begin{array}{l}
\frac{\mu C_{i} W}{L}\left(\left(V_{g}-V_{t h}\right) V_{d s}-\frac{V_{d s}^{2}}{2}\right) \quad \text { for }\left|V_{g}-V_{t h}\right|>\left|V_{d s}\right| \text { (linear regime) } \\
\frac{\mu C_{i} W}{2 L}\left(V_{g}-V_{t h}\right)^{2} \quad \text { for }\left|V_{d s}\right|>\left|V_{g}-V_{t h}\right|>0 \text { (saturation regime) }
\end{array}\right.
$$

where Equation (10) describes the relationship between the drain current $I_{d s}$, the gate-source voltage $V_{g}$ and the drain-source voltage $V_{d s}$ in linear and saturation regimes, respectively. $C_{i}$ is the gate dielectric capacitance per unit area, $\mu$ is the carrier mobility in the semiconductor, $\mathrm{W}$ and $\mathrm{L}$ is the channel width and length of the transistor, respectively. For silicon TFTs, the threshold voltage $V_{t h}$ is defined as the minimum gate-source voltage required to induce strong inversion [59]. However OTFTs and IGZO TFTs usually operate in accumulation region, thus strictly speaking the threshold voltage cannot be defined for OTFTs and IGZO TFTs. Since the threshold voltage concept is nonetheless useful, the compact models will show very different for TFTs with different active materials. Otherwise, the central aim of compact models is to accurately and physically describe the current-voltage characteristics of TFTs in Equation (10). As mentioned above, the surface-potential-based compact model is believed to have high accuracy and strong physical properties. The following will review the surface-potential-based compact models for polysilicon and amorphous silicon TFTs, and then present our compact models for OTFTs and IGZO TFTs based on surface-potential-based.

\subsection{Polysilicon TFT Compact Models}

Polysilicon TFTs have gotten considerable applications, especially in active matrix liquid crystal displays (AMLCDs), printers, scanners, Static Random-Access Memories (SRAMs) and three-dimensional large scale integration (LSI) circuits [60]. In early time, researchers usually built the polysilicon TFT models based on the one-dimensional solution of Poisson's equation and the effects of grain-boundary traps [26,61]. However, these earlier models were unclear for inversion mode devices due to the "reverse" charge shielding concept defined in its derivation [62]. Later, some authors adopted the EMA method to well address the question of non-uniform polysilicon sample with the grain boundaries [29,63]. In 1999, Benjamín et al. also adopted EMA to develop a unified model for long and short-channel polysilicon TFTs [28]. This method is attractive because it accounts for field effect mobility enhancement in the moderate inversion regime and for mobility degradation at high gate voltages, for drain-induced barrier lowering (DIBL) effect, kink effect, off-state current and channel-length modulation. A few years later, Wu et al. proposed a compact model by approximating the generation rate for poly Si TFTs in the leakage region [50]. Although several models for poly-Si TFTs have been proposed so far, based on different equations for the subthreshold, linear, and saturation regions [64,65], these methods always lead to a significant error in evaluating derivatives such as transconductance [66].

To capture more accurate features of poly-Si TFTs, Shimizu et al. developed a compact model based on a new surface-potential-based [67]. Firstly, in the model the states are approximated by the sum of exponential distributions for the deep and tail states as:

$$
g(E)=g_{d e} \exp \left(\frac{E-E_{c}}{E_{d e}}\right)+g_{t a} \exp \left(\frac{E-E_{c}}{E_{t a}}\right)
$$


where $E_{d e}$ and $E_{t a}$ are the inverse slope of deep states and tail states, respectively, $g_{d e}$ and $g_{t a}$ are the density of deep state and tail state at bottom of conduction band $E_{c}$, respectively.

By integrating the 1-D Poisson equation, the surface potentials at the source side as a function of gate voltage can be calculated numerically as the following [68]:

$$
\begin{gathered}
C_{i}\left(V_{g}-V_{f b}-\varphi_{s 0}\right)=\sqrt{\frac{2 q \varepsilon_{s} N_{s u b}}{\beta}}\left[\exp \left(-\beta \varphi_{s 0}\right)-\exp \left(-\beta \varphi_{b 0}\right)+\beta\left(\varphi_{s 0}-\varphi_{b 0}\right)\right. \\
+\left(\frac{n_{i}}{N_{s u b}}\right)^{2}\left[\exp \left(\beta \varphi_{s 0}\right)-\exp \left(\beta \varphi_{b 0}\right)\right]+\left(\frac{\beta N_{\text {deep }}}{\gamma N_{\text {sub }}}\right)\left[\exp \left(\gamma \varphi_{s 0}\right)-\exp \left(\gamma \varphi_{b 0}\right)\right] \\
\left.+\left(\frac{N_{\text {tail }}}{N_{\text {sub }}}\right)\left[\exp \left(\beta \varphi_{s 0}\right)-\exp \left(\beta \varphi_{b 0}\right)\right]\right]^{\frac{1}{2}}
\end{gathered}
$$

where $\gamma=q / E_{d e}, \beta$ is the inverse of thermal voltage, $\varepsilon_{s}$ is the dielectric constant, $N_{\text {sub }}$ is the dopant concentration, $n_{i}$ is the intrinsic carrier concentration, $\varphi_{s 0}$ and $\varphi_{b 0}$ are the front and back surface potentials at the source side, respectively, $N_{\text {deep }}$ and $N_{\text {tail }}$ are the densities of trapped electrons in deep states and tail states under a flat band condition, respectively.

Then, the inversion layer charge density at the source $(x=0)$ or drain $(x=L)$ side can be written as [69] the following:

$$
\begin{aligned}
Q_{i}(x)= & -C_{i}\left(V_{g}-V_{f b}-\varphi_{s 0}\right)+\sqrt{\frac{2 q \varepsilon_{s} N_{s u b}}{\beta}}\left[\exp \left(-\beta \varphi_{s x}\right)-\exp \left(-\beta \varphi_{b x}\right)+\beta\left(\varphi_{s x}-\varphi_{b x}\right)\right. \\
& \left.+\left(\frac{\beta N_{\text {deep }}}{\gamma N_{\text {sub }}}\right)\left[\exp \left(\gamma \varphi_{s x}\right)-\exp \left(\gamma \varphi_{b x}\right)\right]+\left(\frac{N_{\text {tail }}}{N_{\text {sub }}}\right)\left[\exp \left(\beta \varphi_{s x}\right)-\exp \left(\beta \varphi_{b x}\right)\right]\right]^{\frac{1}{2}}
\end{aligned}
$$

Obviously, Equations (12) and (13) could be only solved by iteration. To determine the surface potentials at the source side or at the drain side, the authors used a method from the literature [70]. In Equation (13), the charge densities of inversion layer are derived based on the charge-sheet approximation. Figure 5 shows a comparison of the front surface potentials obtained from Equation (12) and the exact numerical calculations.

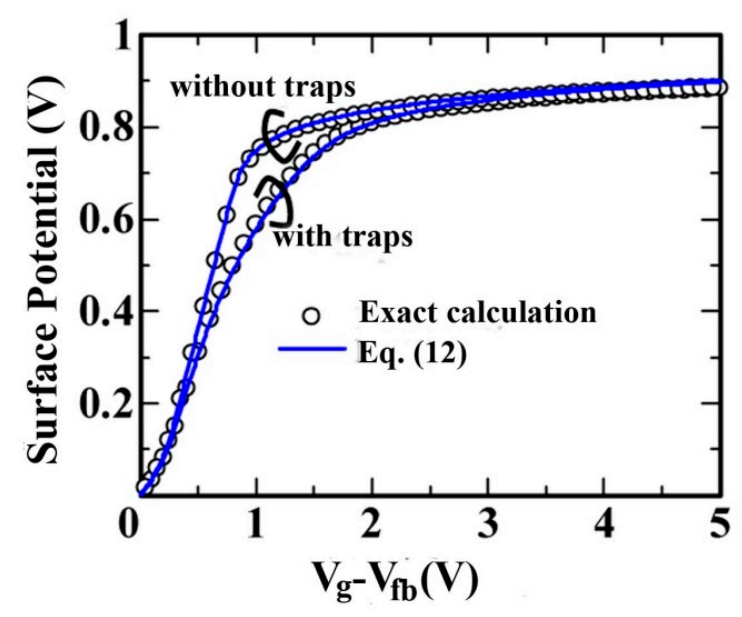

Figure 5. Comparison of calculated front surface potential obtained using Equation (12) (lines) and the exact numerical calculations (circles) with and without traps as a function of gate voltage.

After obtaining the surface potentials, based on the drift-diffusion approximation [71], the authors calculated the drain current as:

$$
\begin{gathered}
I_{d s}=\frac{W \mu}{L \beta}\left[C_{i}\left(\beta\left(V_{g}-V_{f b}\right)+1\right)\left(\varphi_{s L}-\varphi_{s 0}\right)-\frac{\beta}{2} C_{i}\left(\varphi_{s L}^{2}-\varphi_{s 0}^{2}\right)-\frac{\beta}{2}\left(q_{i}(0)+(L)\right)\right. \\
\left.\left(\varphi_{s L}-\varphi_{s 0}\right)-\left(q_{i}(0)-q_{i}(L)\right)\right]
\end{gathered}
$$

where $\varphi_{S L}$ and $\varphi_{b L}$ are the front and back surface potentials at the drain side, respectively, $q_{i}(x)=Q_{i}(x)+C_{i}\left(V_{g}-V_{f b}-\varphi_{s x}\right)$. Note, Equation (14) can describe the drain current in all 
the regions of operation using the unified equation. At the same time, the model did not include the threshold voltage.

Figure 6 shows a comparison of simulated and measured drain current characteristics as a function of gate voltage for an n-channel poly-Si TFT in the subthreshold and above-threshold regions. In the linear and saturation regions, a comparison of simulated and measured drain current characteristics is shown in Figure 7.
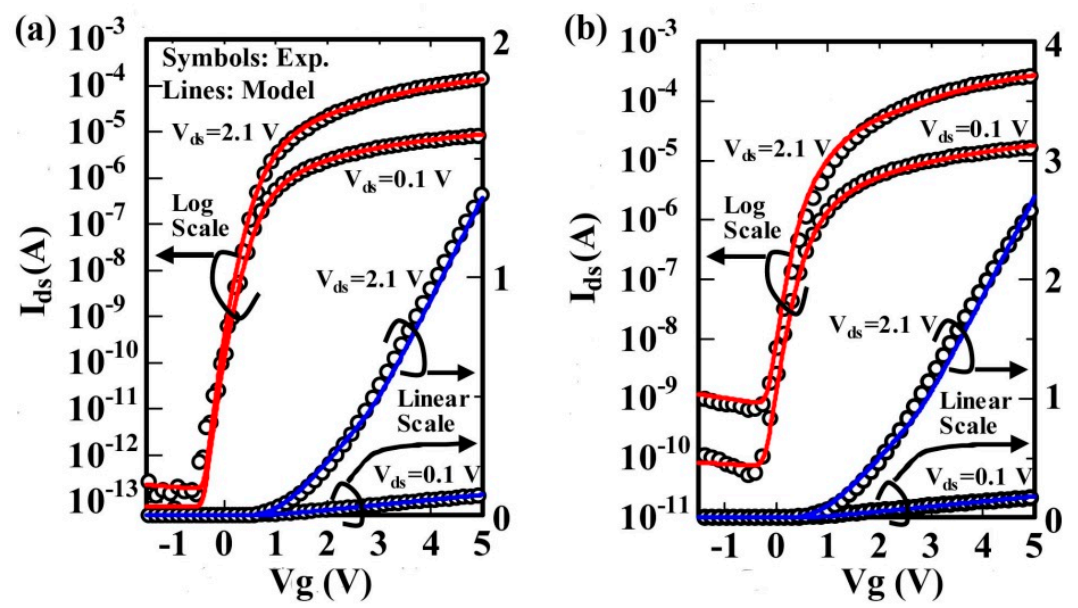

Figure 6. Comparison of measured (circles) and simulated (lines) drain current characteristics as a function of gate voltage on logarithmic (left axis) and linear (right axis) scales for an n-channel poly-Si thin-film transistors (TFT) with (a) W/L $=2 \mu \mathrm{m} / 2 \mu \mathrm{m}$ and (b) $\mathrm{W} / \mathrm{L}=2 \mu \mathrm{m} / 1 \mu \mathrm{m}$.
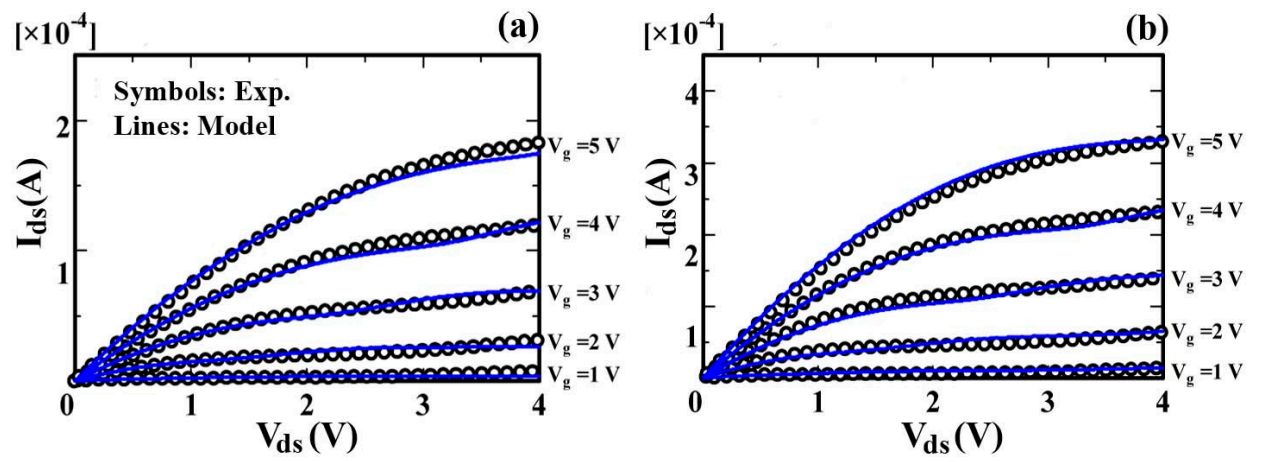

Figure 7. Comparison of measured (circles) and simulated (lines) drain current characteristics as a function of drain voltage for an $n$ channel poly-Si TFT with (a) W/L $=2 \mu \mathrm{m} / 2 \mu \mathrm{m}$ and (b) W/L=2 $\mu \mathrm{m} / 1 \mu \mathrm{m}$. The parameters used in the simulation are the same as those used in Figure 6.

Differing from iterative solution of the surface potential in Shimizu et al.'s model, Chen et al. have developed an analytical solution to the surface potential of poly-Si TFTs by using the Lambert W function [72]. In Chen et al.'s model, the surface potential of poly-Si TFTs can be expressed as,

$$
\left(V_{g}-V_{f b}-\varphi_{s}\right)^{2}=\gamma^{2}\left[\left(1+\frac{N_{T}}{L_{g} N_{A}}\right) \varphi_{s}+\varphi_{t} \exp \left(\frac{\varphi_{s}-\varphi_{n}-2 \varphi_{f}}{\varphi_{t}}\right)\right]-\frac{N_{T}}{L_{g} N_{A}} \varphi_{t} \ln \left(1+K_{m}\right)
$$

where $\varphi_{s}$ is the surface potential, $\gamma$ denotes a body factor: $\gamma=\sqrt{2 \varepsilon_{s} q N_{A}} / C_{i}, L_{g}$ is the grain size, $N_{T}$ and $N_{A}$ are located traps and acceptor density, respectively, $\varphi_{t}$ is the thermal voltage, $\varphi_{f}$ is the Fermi potential, $\varphi_{n}$ is the channel voltage, $K_{m}=0.5 \exp \left(\frac{E_{t}+q \varphi_{t}}{k_{B} T}\right)$. To derive an analytical and non-iterative evaluation, the normalized form of Equation (15) can be written as the following:

$$
\left(v_{g}-x_{W}\right)^{2}=G_{T F T}^{2}\left[x_{W}+\Delta_{T F T} \exp \left(x_{W}\right)+A\right]
$$


where $x_{W}=\varphi_{s} / \varphi_{t}$ is the normalized surface potential, $v_{g}=\left(V_{g}-V_{f b}\right) / \varphi_{t}$ the normalized effective gate voltage, $G_{T F T}=\gamma \sqrt{\frac{1+N_{T} / L_{g} N_{A}}{\varphi_{t}}}, \Delta_{T F T}=\exp \left(\frac{-\varphi_{t}-2 \varphi_{f}}{\varphi_{t}}\right) /\left(1+\frac{N_{T}}{L_{g} N_{A}}\right)$, and $A=-\frac{N_{T} \ln \left(1+K_{m}\right)}{N_{T}+L_{g} N_{A}}$.

Then, with a simple mathematical procedure and using the principal branch of the Lambert $\mathrm{W}$ function [73], the authors obtained the physics-based analytical solution of the normalized surface potential as follows:

$$
x_{W}=-W_{0}\left[f \times \Delta_{T F T} \exp \left(v_{G}-f \times A\right)\right]+v_{G}-f \times A
$$

where $v_{G}=\left(v_{g}+\frac{G_{T E T}^{2}}{2}\right)-G_{F E T} \sqrt{v_{g}+G_{T F T}^{2} / 4}$ and $f=G_{F E T} / 2 \sqrt{v_{g}+G_{T F T}^{2} / 4}$.

In order to improve the accuracy, some corrections by using the Schroder series in the surface potential expression have been provided for Equation (17). Finally, the complete solution to the physics-based surface potential of poly-Si TFTs with absolute error only in nanovolt range can be expressed as:

$$
\varphi_{s}=\left[x_{W}+\omega\left(y_{W}, y_{W}^{\prime}, y_{W}^{\prime \prime}\right)+\varepsilon\right] \varphi_{t}
$$

Based on Equation (18), the surface potential derivative with respect to the gate voltage has been calculated [72], as shown in Figure 8. Figure 8 shows that no splits and peaks exist near the flatband regions, which suggests that the analytical solution to the surface potential is better than the algorithm in the Penn State Philips (PSP) model [74].

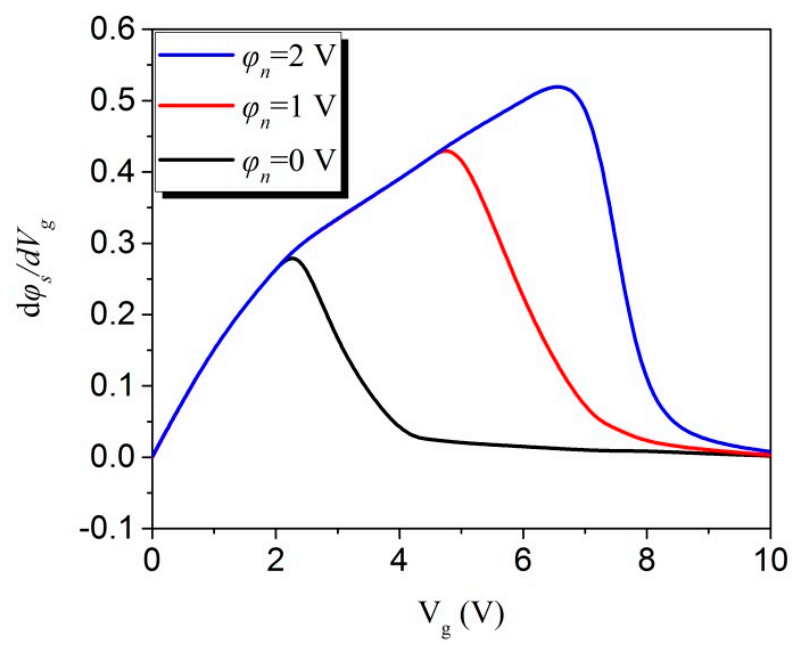

Figure 8. The characteristics of the surface potential derivative with respect to the gate voltage for different channel potential $\varphi_{n}$.

Based on the formulas of surface potential from Chen et al., subsequently some researchers presented a complete modeling for surface potential in partially depleted poly-Si TFTs with undoped or lightly doped body by including both monoenergetic and exponential trap distributions [32]. The proposed closed-form algorithm is able to accurately calculate the surface potential and has the advantage of both accuracy and computational efficiency, which is useful for compact modeling and CAD applications.

\subsection{Amorphous Silicon TFTs}

Amorphous Silicon, especially hydrogenated amorphous-silicon (a-Si:H), has been considered as the most well-studied materials for TFTs. Generally speaking, the most important features of amorphous silicon TFT characteristics can be described by analyzing the device behavior in two regimes: below-threshold, when the electron quasi-Fermi level is in the deep states; and above-threshold, when the Fermi level enters the tail states [75]. A current model for the 
below- and above-threshold regimes had been proposed by considering the sheet carrier density as a function of Fermi lever position by Shur et al. [76]. In 1997, Shur et al. again developed a physically based analytical model for n-channel amorphous silicon thin film transistors and for $\mathrm{n}$ and p-channel polysilicon thin film transistors, which covered all regimes of transistor operation: leakage, subthreshold, above-threshold conduction, and the kink regime in polysilicon thin film transistors [63]. Only in the last few years several models have been built, based on the description of below-threshold and above-threshold, respectively [77-79]. However, with gradual accumulation of the requirements imposed on the compact models and simultaneous realization of the limitations associated with the traditional modeling techniques, new physical phenomena become essential for the accurate reproduction of the device characteristics. On the other hand, due to these drawbacks in the regional approach, analytical models based on surface potential have been paid more attentions in the development of device models [40].

In terms of the consideration above, in 2008, Liu et al. presented an analytical a-Si:H TFTs model based on the surface potential [80]. In the model, when TFT is biased, the majority of the induced charges in the channel are trapped in the acceptor-like states, which divided into two groups: deep states and tail states. The distribution of localized acceptor states can be expressed as Equation (11). The localized trapped charge density is expressed as:

$$
n_{\text {trapped }}=\int_{-\infty}^{E_{c}} \frac{g(E)}{1+\frac{1}{g} \exp \left(\frac{E-E_{f}}{k_{B} T}\right)}
$$

here $g$ is the degenerescence factor of localized states. When the density of trapped charges in the tail states are considered, the integral (Equation (19)) can be rewritten as [68] the following:

$$
n_{\text {tail }}=g_{t} g^{T / T_{0}} \frac{k_{B} T}{q} f\left(T, T_{t}\right) \exp \frac{q \varphi-q V_{c h}(y)-E_{f 0}}{k_{B} T_{0}}
$$

where $g_{t}$ is the tail states density at $E_{c}, T_{t}$ is the tail state characteristic temperature, $V_{c h}$ stands for the channel quasi-Fermi level which is the channel voltage equal to 0 at the source and $V_{d s}$ at the drain.

To obtain the potential, the authors then solved the Poisson's equation:

$$
\frac{\partial^{2} \varphi}{\varphi x^{2}}=-\frac{d F}{d x}=\frac{q}{\varepsilon_{s}}\left(n_{\text {deep }}+n_{\text {tail }}+n_{\text {free }}\right)
$$

where $n_{\text {deep }}, n_{\text {tail }}$ and $n_{\text {free }}$ are the densities for deep trap, free, tail trap charges, respectively, $n_{\text {free }}=N_{c} \exp \left(\frac{q \varphi_{s}-q V_{c h}(y)-E_{f 0}}{k_{B} T}\right)$. According to Gauss' law, and introducing electrical field effect, the relationship between the gate-source voltage and the surface potential can be found as follows

$$
\begin{aligned}
& C_{i}\left(V_{g}-V_{f b}-\varphi_{s}\right)=r_{t} \varepsilon_{s} \exp \left(\frac{q \varphi_{s}-q V_{c h}(y)-E_{f 0}}{k_{B} T_{0}}\right)+r_{d} \varepsilon_{s} \exp \left(\frac{q \varphi_{s}-q V_{c h}(y)-E_{f 0}}{k_{B} T_{d}}\right) \\
& +r_{f} \varepsilon_{s} \exp \left(\frac{q \varphi_{s}-q V_{c h}(y)-E_{f 0}}{k_{B} T}\right)
\end{aligned}
$$

here $r_{t}=\sqrt{\frac{2 k_{B} T_{0} g_{t} g^{T / T_{0}} f\left(T, T_{t}\right) k_{B} T}{q \varepsilon_{s}}}, r_{d}=\sqrt{\frac{2 k_{B} T_{d} g_{d} g^{T / T_{d}} \pi k_{B} T}{q \varepsilon_{s} \sin \left(\frac{\pi T}{T_{d}}\right)}}$ and $r_{f}=\sqrt{\frac{2 k_{B} T N_{c}}{\varepsilon_{s}}}$.

To derive analytical and noniterative evaluation from Equation (22), the normalized form of Equation (22) can be written as follows:

$$
(x g-x)=G_{t} \exp (x-x n)+G_{d}[\exp (x-x n)]^{T_{0} / T_{d}}+G_{f}[\exp (x-x n)]^{T_{0} / T}
$$

where $x g=-\frac{V_{g}-V_{f b}}{2 V_{t o}}, x=\frac{\varphi_{s}}{2 V_{t o}}, x n=\frac{\frac{E_{f 0}}{q}+V_{c h}(y)}{2 V_{t o}}, G_{t}=\frac{r_{t} \varepsilon_{s}}{2 C_{i} V_{t o}}, G_{d}=\frac{r_{d} \varepsilon_{s}}{2 C_{i} V_{t o}}, G_{f}=\frac{r_{f} \mathcal{E}_{s}}{2 C_{i} V_{t o}}$, and $V_{t o}=\frac{k_{B} T_{o}}{q}$. 
Then, by using the two-order Taylor expansion, the solution for the surface potential of amorphous silicon TFTs is expressed by:

$$
\varphi_{s}=x \cdot 2 V_{t o} .
$$

Based on the solution for the surface potential of amorphous silicon TFTs, the authors compared the analytical results with the numerical results, as shown in Figure 9a. And the absolute errors of the new analytical approximation were shown in Figure $9 \mathrm{~b}$. The absolute errors introduced by analytical approximation are less than $0.02 \mathrm{~V}$ in all cases.
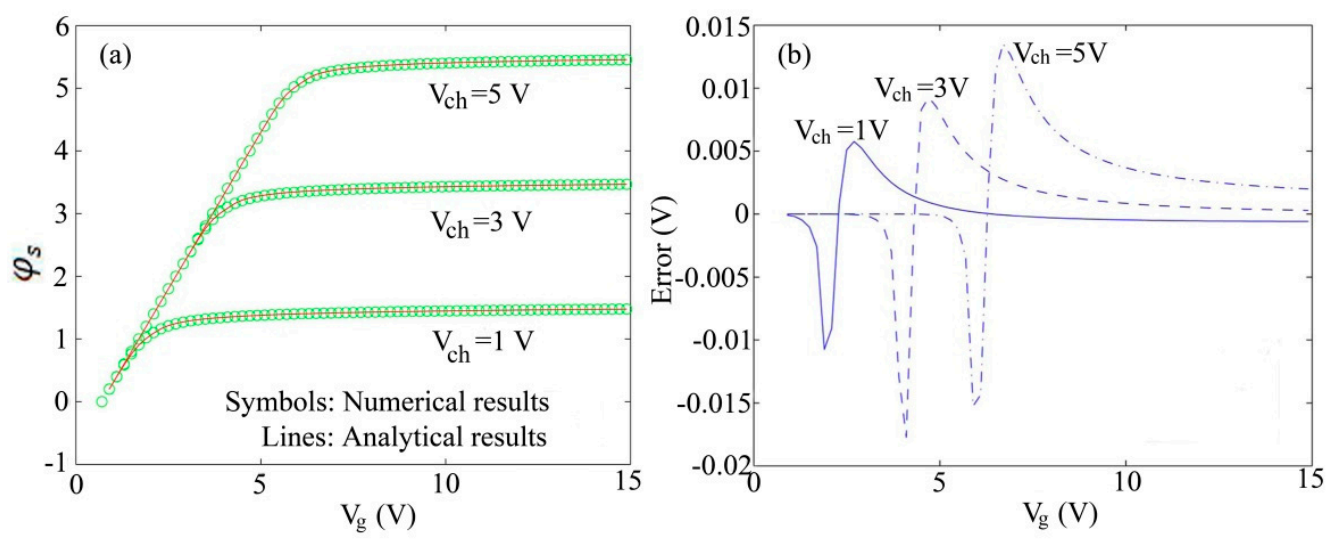

Figure 9. (a) Comparison of analytical results with the numerical results; and (b) absolute error of the new analytical approximation for the surface potential.

After the surface potential is solved precisely, the authors then discussed the drain current by dividing the new derivation of the DC model into the below threshold region and the above threshold region.

Below threshold region, the static current of amorphous TFTs is written as:

$$
\begin{aligned}
& I_{d s d}=\mu_{n} \frac{W}{L} N_{c} \varepsilon_{s} \frac{2 k_{B} T_{d} k_{B} T}{2 k_{B} T_{d}-k_{B} T}\left(\frac{1}{r_{d} \varepsilon_{s}}\right)^{\frac{2 T_{d}}{T}} C_{i}^{\frac{2 T_{d}}{T}-1}\left[\frac{T}{2 T_{d}}\left(\Delta \varphi_{s s}^{\frac{2 T_{d}}{T}}-\Delta \varphi_{s d}^{\frac{2 T_{d}}{T}}\right)\right. \\
& \left.+2 V_{t d} \frac{T}{2 T_{d}-T}\left(\Delta \varphi_{s s}^{\frac{2 T_{d}}{T}-1}-\Delta \varphi_{s d}^{\frac{2 T_{d}}{T}-1}\right)\right]
\end{aligned}
$$

Above threshold region, similarly, the expression of drain current in the above threshold regime can be obtained as:

$$
\begin{aligned}
& I_{d s t}=\mu_{n} \frac{W}{L} N_{c} \varepsilon_{s} \frac{2 k_{B} T_{0} k_{B} T}{2 k_{B} T_{0}-k_{B} T}\left(\frac{1}{r_{t} \varepsilon_{s}}\right)^{\frac{2 T_{0}}{T}} C_{i}^{\frac{2 T_{0}}{T}-1}\left[\frac{T}{2 T_{0}}\left(\Delta \varphi_{s S}^{\frac{2 T_{0}}{T}}-\Delta \varphi_{s d}^{\frac{2 T_{0}}{T}}\right)\right. \\
& \left.+2 V_{t 0} \frac{T}{2 T_{0}-T}\left(\Delta \varphi_{s s}^{\frac{2 T_{0}}{T}-1}-\Delta \varphi_{s d}^{\frac{2 T_{0}}{T}}-1\right)\right]
\end{aligned}
$$

According to the expression of drain current, the calculated transfer characteristics for a-Si:H TFT is shown in Figure 10a. It is noted that a smooth transition is achieved in the below- and above-threshold regions without any use of smooth functions. Furthermore, the threshold voltage is not required in the whole calculations. Figure $10 \mathrm{~b}$ displays the measured characteristics and the calculated current-voltage characteristics of an a-Si:H TFT. It is demonstrated that the model exhibits a reasonable agreement in both the linear region and the saturation region. 
(a)

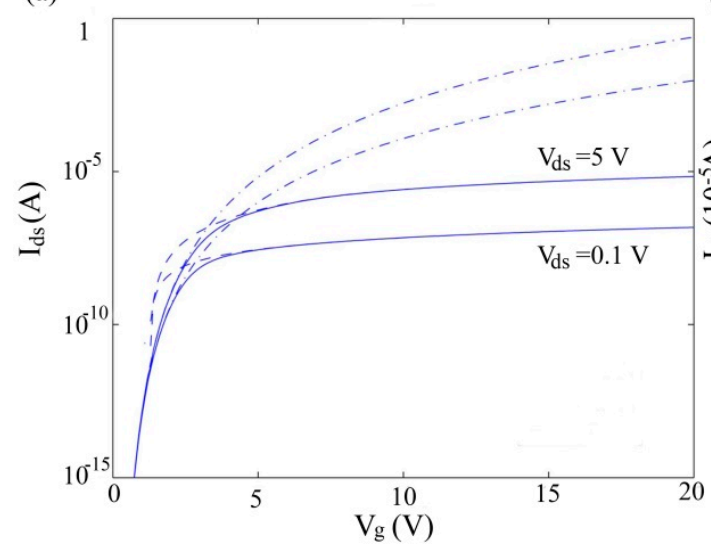

(b)

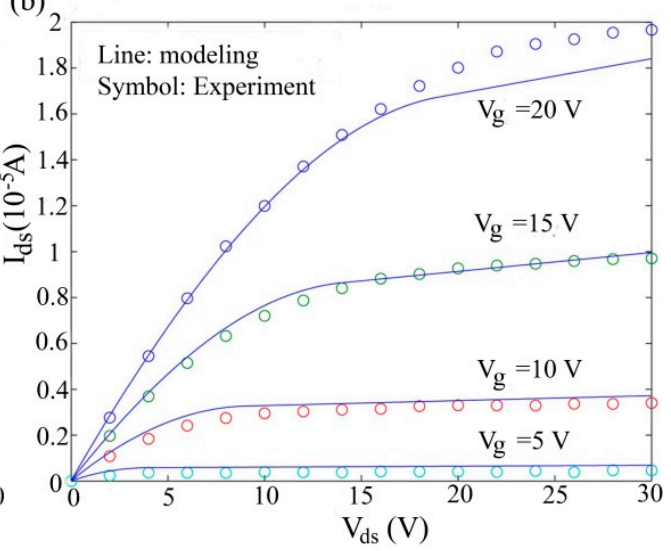

Figure 10. (a) Calculated transfer characteristics and (b) calculated output characteristics for a-Si:H TFT, with the measured data for comparison.

To calculate the surface potential, other methods have been used. For example, very recently, Qin et al. developed a novel scheme for surface potential of amorphous silicon TFTs by taking deep Gaussian and tail exponential distribution of the density of states into account [81]. In Qin et al.'s model, the authors adopted Taylor expansion below threshold regime, and the principle of Lamber W function and Schroder series above threshold regime, as well as Chen et al.'s model in Section 3.1.

\subsection{OTFT Compact Models}

In OTFTs, the energy disorder is usually described by Gaussian DOS as [82]:

$$
g(E)=\frac{N_{t}}{\sqrt{2 \pi} \sigma} \exp \left(-\frac{E^{2}}{2 \sigma^{2}}\right)
$$

where $N_{t}$ is the total localized states, and $\sigma$ indicates the width of the DOS. By connecting Gauss law $C_{i}\left(V_{g}-V_{f b}-\varphi_{s}\right)=\varepsilon_{s} F(0)$, one can obtain the following:

$$
C_{i}\left(V_{g}-V_{f b}-\varphi_{s}\right)=\sqrt{\frac{2 q \varepsilon_{s} N_{t}}{\sqrt{2 \pi} \sigma} \int_{0}^{\varphi_{s}} \int_{-\infty}^{\infty} \frac{\exp \left(-E^{2} / 2 \sigma^{2}\right)}{1+\exp \left(\frac{E-E_{f 0}-q(\varphi-V)}{k_{B} T}\right)} d E d \varphi}
$$

where $F(0)$ is the electric field perpendicular to the interface at the interface, $V$ is the channel voltage, and $E_{f 0}$ is the Fermi level far from the semiconductor-insulator interface.

By approximating the Fermi-Dirac distribution with the Boltzmann distribution, Equation (28) can be rewritten as:

$$
C_{i}\left(V_{g}-V_{f b}-\varphi_{s}\right)=\sqrt{\frac{2 q \varepsilon_{s} N_{t}}{\sqrt{2 \pi} \sigma} \int_{0}^{\varphi_{s}} \int_{-\infty}^{\infty} \exp \left(-\frac{E^{2}}{2 \sigma^{2}}-\frac{E-E_{f 0}-q(\varphi-V)}{k_{B} T}\right) d E d \varphi}
$$

Since the localized states mainly lie in the higher energy of Gaussian DOS, $E-E_{f 0}>2 k_{B} T$ is usually achieved. As the carrier density varies over a narrow range, then the Fermi-Dirac distribution can be approximated by the Boltzmann distribution. According to Equation (29), the surface potential can be calculated as:

$$
\left(V_{g}-V_{f b}-\varphi_{s}\right)^{2}=k \exp \left(-\frac{V}{\varphi_{t}}\right)\left(\exp \left(\frac{\varphi_{s}}{\varphi_{t}}\right)-1\right)
$$


where $k=\frac{\varepsilon_{0} \varepsilon_{s} k_{B} N_{t}}{C_{i}^{2}} \exp \left(-0.5 \sigma^{2}\right)$. The solution of Equation (30) actually is numerical. However, under low gate voltage OTFTs operate in weak accumulation mode, that is, $\varphi_{s} \ll \varphi_{t}$. In this situation, the surface potential $\varphi_{s w}$ is small and can be obtained as

$$
\phi_{s w}=V_{g}-V_{f b}+\frac{k \exp \left(-\frac{V}{\varphi_{t}}\right)}{2 \varphi_{t}}-\sqrt{\left(V_{g}-V_{f b}+\frac{k \exp \left(-v / \varphi_{t}\right)}{2 \varphi_{t}}\right)^{2}-\left(V_{g}-V_{f b}\right)}
$$

Under high gate voltage, OTFTs operate in a strong accumulation mode, that is, $V_{g}-V_{f b} \gg \varphi_{s} \gg$ $\varphi_{t}$. In this case, the surface potential $\varphi_{s s}$ reads as

$$
\phi_{s s}=2 \varphi_{t} \ln \left(\frac{V_{g}-V_{f b}}{\sqrt{k}}\right)+V
$$

Connecting Equations (31) and (32), the unified surface potential of OTFTs is expressed as

$$
\varphi_{s}=\sqrt{\frac{\varphi_{s w}^{\gamma} \cdot \varphi_{s s}^{\gamma}}{\varphi_{s w} \gamma+\varphi_{s s} \gamma}}
$$

Figure 11a shows the comparison between the surface potential calculated using the Boltzmann distribution and Fermi-Dirac distribution functions under different channel voltages, respectively [83]. One can see that a good agreement is observed. Figure $11 \mathrm{~b}$ shows the absolute and relative error of the Boltzmann function approximation from Figure 11a, revealing that the maximum of relative error is less than $0.6 \%$, as shown by the maximum peak in Figure $11 \mathrm{~b}$. This approximation displays good accuracy for weak, moderate and strong accumulation at various channel voltages. Otherwise, the absolute error of the surface potential introduced by the Boltzmann function approximation decreases with channel voltage and is always lower than $0.035 \mathrm{~V}$.

(a)

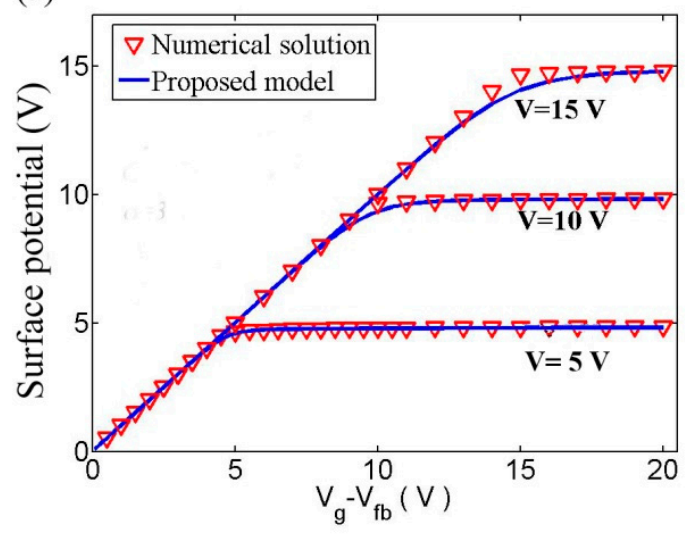

(b)

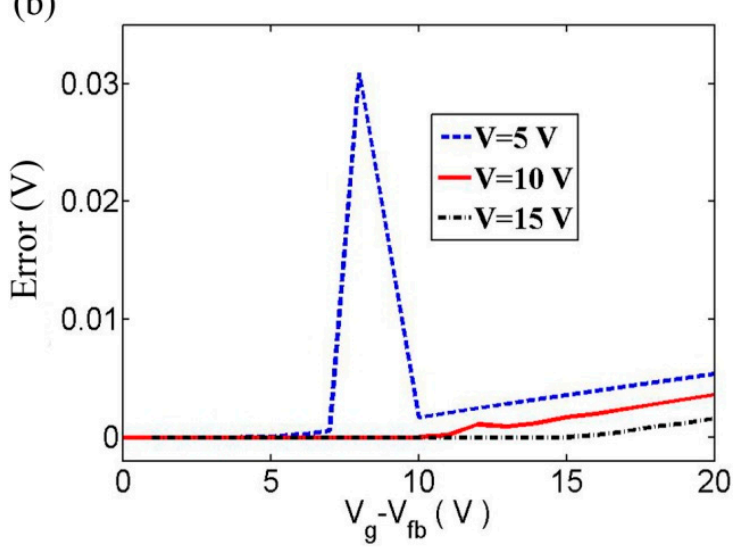

Figure 11. (a) Comparison between the surface potential calculated using Boltzmann distribution and Fermi-Dirac distribution functions for different channel voltages and (b) absolute error of the Boltzmann approximation from (a).

For OTFTs, the field-effect mobility can be written as [84]:

$$
\mu=\mu_{0} \exp \left(C_{1}\left(2 n / N_{t}\right)^{C_{2}}\right)
$$

here $C_{1}$ and $C_{2}$ are given as $C_{1}=0.5\left(S^{2}-S\right)$ and $C_{2}=2 \frac{\ln \left(S^{2}-S\right)-\ln (\ln (4))}{S^{2}}$, which only depend on the disorder, $n$ is the carrier concentration, $\mu_{0}=\mu_{00} \exp \left(-a S-b S^{2}\right), S=\sigma / k_{B} T$, and $\mu_{00}$ is the mobility in the limit $n \rightarrow 0$. 
Using the same method in the literature [85], the field-effect mobility $\mu_{e f f}$ is calculated with the following:

$$
\begin{gathered}
\mu_{e f f}=\frac{L}{C_{i} W V_{d s}} \frac{\partial I_{d s}}{\partial V_{g}}=\frac{\sqrt{\varepsilon_{s} \varepsilon_{0} / 2 q}}{C_{i}} \frac{n\left(\varphi_{s}\right) \mu\left(n\left(\varphi_{s}\right)\right)}{\sqrt{\int_{0}^{\varphi} \int_{-\infty}^{\infty} g(E) d E d \varphi}} \times \frac{2 C_{i} \sqrt{\int_{0}^{\varphi_{s}} \int_{-\infty}^{\infty} g(E) d E d \varphi}}{\sqrt{\varepsilon_{s} q n}\left(\varphi_{s}\right)} \\
\approx \mu_{0} \exp \left(C_{1} \frac{\left(2 C_{i}\right)^{2 C_{2}}}{\left(2 \varepsilon_{s} k_{B} T N_{t}\right)^{C_{2}}}\left(V_{g}-V_{f b}-\gamma V_{d s}\right)^{2 C_{2}}\right)
\end{gathered}
$$

where $n\left(\varphi_{s}\right)=\int_{-\infty}^{\infty} g(E)\left(1+\exp \left(\frac{E-E_{f 0}-q(\varphi-V)}{k_{B} T}\right)\right)^{-1} d E, \gamma$ is a parameter that accounts for channel-length modulation.

Then, according to Gauss's law, the sheet density of total induced charges in the channel is given by:

$$
Q_{i}=C_{i}\left(V_{g}-V_{f b}-\phi_{s}\right) \approx C_{i} \sqrt{K} \exp \left(\frac{\left(\varphi_{s}-V\right)}{2 \varphi_{t}}-1\right)
$$

By differentiating Equation (36) with respect to $\varphi_{s}$, we then obtain:

$$
\frac{d V}{d \varphi_{s}}=\frac{2 \varphi_{t}}{\sqrt{K}} \exp \left(-\frac{\varphi_{s}-V}{2 \varphi_{t}}+1\right)+1=2 \varphi_{t} \frac{C_{i}}{Q_{i}}+1
$$

Using the gradual channel approximation, $I_{d s}$ is given by:

$$
I_{d s}=-\mu_{e f f} W Q_{i} \frac{d V}{d y}=-\mu_{e f f} W Q_{i}\left(2 \varphi_{t} \frac{C_{i}}{Q_{i}}+1\right) \frac{d \varphi}{d y}
$$

By integrating Equation (38) from $\varphi_{s}=\varphi_{s s}$ to $\varphi_{s}=\varphi_{s d}$, the static current of OTFTs becomes:

$$
I_{d s 0}=\frac{\mu_{e f f} W}{L}\left(2 \varphi_{t} C_{i}\left(\varphi_{s d}-\varphi_{s s}\right)-\frac{C_{i}}{2}\left(\left(V_{g}-V_{f b}-\varphi_{s d}\right)^{2}-\left(V_{g}-V_{f b}-\varphi_{s s}\right)^{2}\right)\right)
$$

where $\varphi_{s s}$ and $\varphi_{s d}$ are the surface potentials at the source and drain side, respectively. Both of them can be analytically calculated by Equation (33). When OTFTs are biased to the saturation region, channel-length modulation becomes significant in short channel devices. In this case, the expression of $I_{d s}$ can be rewritten as:

$$
I_{d s}=I_{d s 0}\left(1+\lambda V_{d s}\right)
$$

Based on Equation (40), the OTFT characteristics can be described by a new formula that does not contain the threshold voltage.

Figure 12 shows the measured characteristics from pentacene transistors and the calculated current-voltage characteristics of OTFT. The model agrees well with the experimental results in both the linear and saturation regions [83]. 
(a)

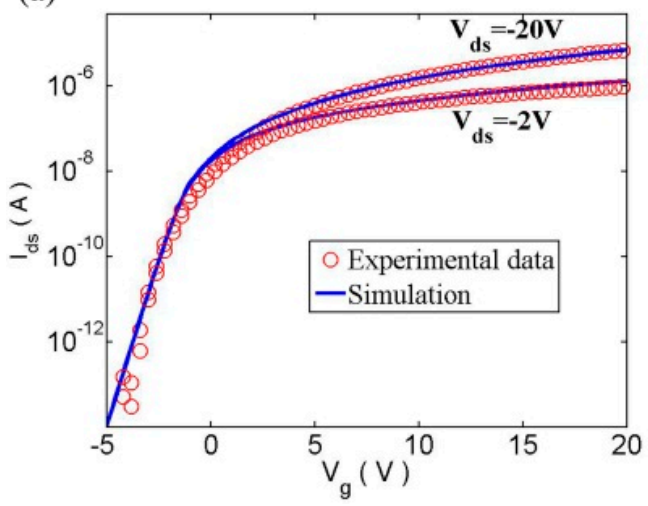

(b)

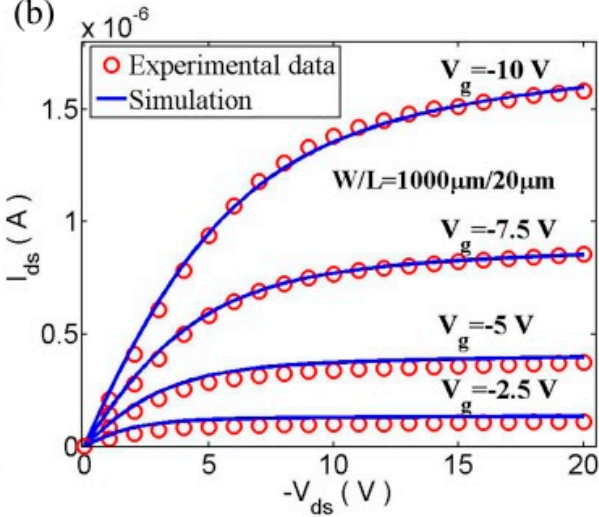

Figure 12. (a) Simulated and experimental results for transfer characteristics of organic Thin-film transistors (OTFT); and (b) comparison between the simulated and experimental results for output characteristics of OTFT for different gate voltages.

We also verified our proposed model by comparing it to measurements of OTFTs with channel lengths from $25 \mu \mathrm{m}$ to $5 \mu \mathrm{m}(W=1000 \mu \mathrm{m})$, as shown in Figure 13 [83]. The extracted $\lambda$ values are 0.55 and 0.27 for $L=5 \mu \mathrm{m}$ and $L=10 \mu \mathrm{m}$, respectively.
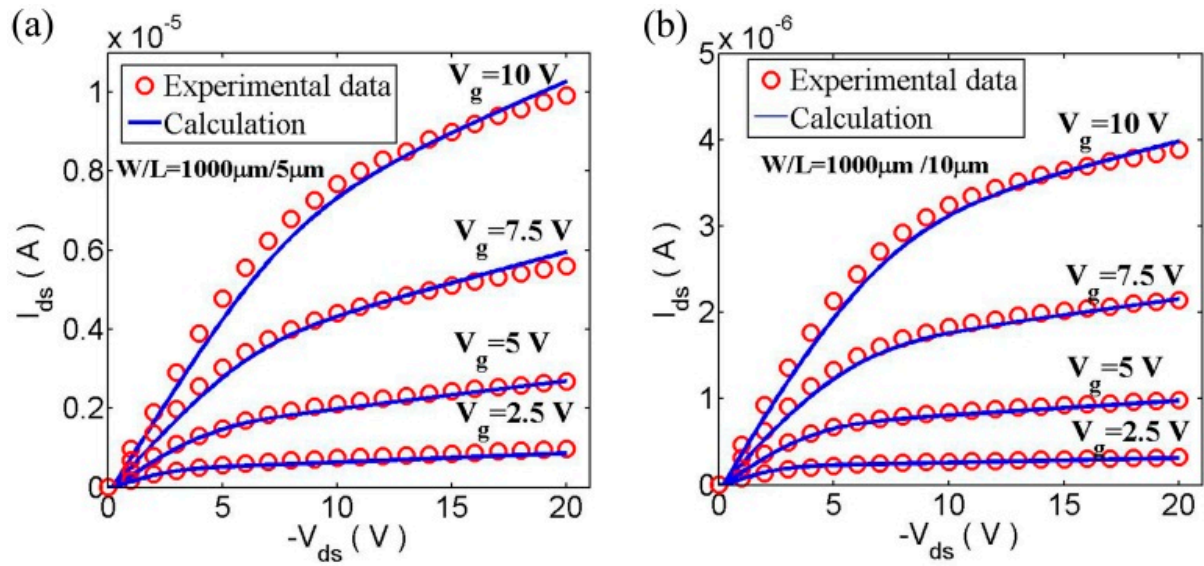

Figure 13. Simulated and experimental results of output characteristics of OTFT: (a) for $\mathrm{W} / \mathrm{L}=1000 \mu \mathrm{m}$ $/ 5 \mu \mathrm{m}$ and $(\mathbf{b})$ for $\mathrm{W} / \mathrm{L}=1000 \mu \mathrm{m} / 10 \mu \mathrm{m}$.

\section{4. a-IGZO TFTs}

As mentioned in Section 2.3, the MTR theory is responsible for the charge transport of a-IGZO TFTs. We have combined the MTR theory with the surface potential to develop the compact model of IGZO TFTs [86,87]. Generally speaking, in TFTs, due to the accumulated carriers in semiconductor-insulator interface under the gate voltage, the gate-induced potential $\varphi(x)$ shifts the difference between the mobility edge and the Fermi level. The quasi-Fermi level $E_{f}(x)$ is

$$
E_{f}(x)=E_{f 0}+q \varphi(x)
$$

The variation of $\varphi(x)$ with respect to the distance $x$ is determined by the Poisson equation as [57]:

$$
F(x)^{2}=\frac{2 q}{\varepsilon_{s}} n_{\text {total }}=\frac{2 q}{\varepsilon_{S}}\left[N_{t} v_{0} \tau_{0} \exp \left(\frac{E_{f}(x)}{k_{B} T}\right)+\int_{0}^{\varphi(x)} \int_{-\infty}^{0} \frac{g(E)}{1+\exp \left(\frac{E-E_{f}(x)}{k_{B} T}\right)} d E d \varphi(x)\right]
$$


where $F(x)$ is the electric field perpendicular to the interface. At the interface, the electric field $F(0)$ can be expressed through Gauss's law as:

$$
\varepsilon_{s} F(0)=C_{i}\left(V_{g}-V_{f b}-\varphi_{s}\right)=\sqrt{2 q \varepsilon_{s}\left[N_{t} v_{0} \tau_{0} \exp \left(\frac{E_{f}(x)}{k_{B} T}\right)+\int_{0}^{\varphi(x)} \int_{-\infty}^{0} \frac{g(E)}{1+\exp \left(\frac{E-E_{f}(x)}{k_{B} T}\right)} d E d \varphi(x)\right]}
$$

where $T_{T A}$ is the characteristic temperature of the exponential DOS, $\tau_{0}$ is the lifetime of carriers, and $v_{0}$ is the attempt-to-escape frequency. Then, the field effect mobility could be written as [88]:

$$
\mu_{e f f}=\frac{\mu_{e}}{1+\left(\frac{1}{v_{0} \tau_{0}} \Gamma\left(1+T / T_{T A}\right) \Gamma\left(1-T / T_{T A}\right) \exp \left(\frac{E_{f 0}+q \varphi_{s}}{k_{B} T_{T A}}\left(\frac{T}{T_{T A}}-1\right)\right)\right)^{-1}}
$$

where $\mu_{e}$ is the band mobility and $\Gamma(z)=\pi z / \sin \pi z$. Under the low gate voltage, Fermi level lies in the deep states and hence free carriers above the mobility edge can be neglected, and carriers of localized states will dominate the transport of IGZO TFTs (corresponding to the sub-threshold regime of transistor). Thus, the total carrier concentration is reasonably written as

$$
n(x) \approx \int_{-\infty}^{0} \frac{g(E, x)}{1+\exp \left(\frac{E-E_{f}(x)}{k_{B} T}\right)} d E=N_{t} \Gamma\left(1+\frac{T}{T_{T A}}\right) \Gamma\left(1-\frac{T}{T_{T A}}\right) \exp \left(\frac{E_{f 0}+q \varphi_{s}}{k_{B} T_{T A}}\right)
$$

Substituting Equations (45) into (43), one can get the following expression:

$$
C_{i}\left(V_{g}-V_{f b}-\varphi_{s}\right)=\sqrt{q \mathcal{E}_{s}\left[N_{t} v_{0} \tau_{0} \exp \left(\frac{E_{f}(x)}{k_{B} T}\right)+N_{t} \Gamma\left(1+\frac{T}{T_{T A}}\right) \Gamma\left(1-\frac{T}{T_{T A}}\right) \exp \left(\frac{E_{f 0}+q \varphi_{s}}{k_{B} T_{T A}}\right)\right]}
$$

To achieve the analytic solution of the surface potential, we transformed Equation (46) as:

$$
V_{g}-V_{f b}-\varphi_{s}=G_{T} \exp \left(\frac{q \varphi_{s}-q V_{c h}}{2 k_{B} T}\right)+G_{T A} \exp \left(\frac{q \varphi_{s}-q V_{c h}}{2 k_{B} T_{T A}}\right)
$$

$G_{T}$ and $G_{T A}$ can be expressed as:

$$
\left\{\begin{array}{l}
G_{T}=\frac{1}{C_{i}} \sqrt{q \varepsilon_{s} v_{0} \tau_{0} N_{t} \exp \left(\frac{E_{f 0}}{k_{B} T}\right)} \\
G_{T A}=\frac{1}{C_{i}} \sqrt{q \varepsilon_{s} N_{t} \Gamma\left(1+\frac{T}{T_{T A}}\right) \Gamma\left(1-\frac{T}{T_{T A}}\right) \exp \left(\frac{E_{f 0}}{k_{B} T_{T A}}\right)}
\end{array}\right.
$$

Through estimating the order of magnitudes, in Equation (47) the first term is much smaller than the second term. Thus, we only consider the second term and ignore the first term. By using two-order Taylor expansion, one can get:

$$
x_{i}=x g\left\{\left[(x g+1)^{2}+2 x n+2 \log \left(\frac{x g}{G_{T}}\right)\right]^{1 / 2}-x g-1\right\}
$$

However, if one considers only the second term in Equation (47), some errors in the surface potential calculation maybe occur. In order to improve the accuracy, we add some corrections by using the Schroder series method to cover the influence of the first term in Equation (47). Finally, the analytical solution of the surface potential can be written as:

$$
\left\{\begin{array}{l}
\varphi_{s}=2 \frac{k_{B} T_{T A}}{q}\left[x_{i}-\frac{f}{\partial f}\left(1+\frac{\partial^{2} f}{2 \partial f} \frac{f}{\partial f}\right)\right] \\
f=(x g-x)-G_{T A} \exp (x-x n)-G_{T}(\exp (x-x n))^{\frac{T_{T A}}{T}}
\end{array}\right.
$$


Figure 14 shows a comparison of calculated surface potential between analytic solution and numerical result $[86,87]$. The percentage error between the numerical and analytical solutions is always below $0.2 \%$. The parameters are $T=300 \mathrm{~K}, T_{T A}=405 \mathrm{~K}, v_{0} \tau_{0}=1, V_{f b}=0.5 \mathrm{~V}, C_{i}=8.85 \times 10^{-8} \mathrm{~F} / \mathrm{cm}^{2}$, and $\mu_{e}=19.7 \mathrm{~cm}^{2} / \mathrm{Vs}$.

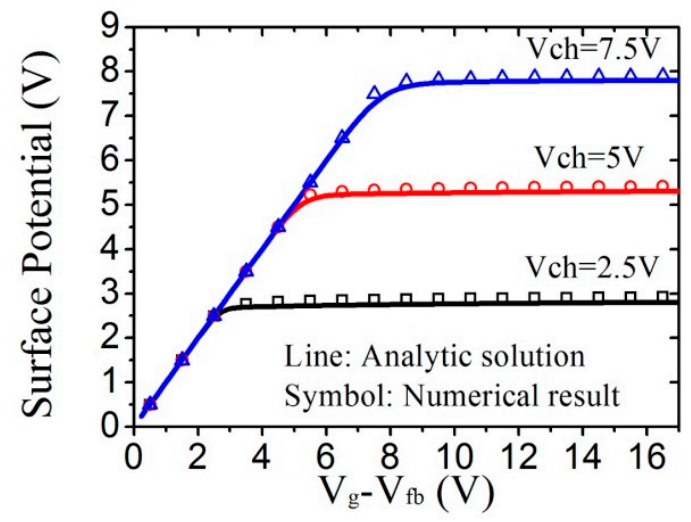

Figure 14. Comparison of the calculated surface potential between analytic solution and the numerical results for different channel voltages.

Using the gradual channel approximation, the current equation is given as:

$$
I_{d s}=-\mu_{e f f} W Q_{i} \frac{d V}{d y}=-\mu_{e f f} W Q_{i}\left(2 \varphi_{t} \frac{C_{i}}{Q_{i}}+1\right) \frac{d \varphi}{d y}
$$

By integrating Equation (51) from $\varphi_{s}=\varphi_{s s}$ to $\varphi_{s}=\varphi_{s d}$, the static current of a-IGZO TFTs is expressed as:

$$
I_{d s 0}=\mu_{e f f} \frac{W}{L}\left[2 \varphi_{t} C_{i}\left(\varphi_{s d}-\varphi_{s s}\right)-\frac{1}{2}\left(\left(V_{g}-V_{f b}-\varphi_{s d}\right)^{2}-\left(V_{g}-V_{f b}-\varphi_{s s}\right)^{2}\right)\right]
$$

where $\varphi_{s s}$ and $\varphi_{s d}$ are the surface potential at source and drain side, respectively. Both of them can be analytically calculated from Equation (50).

Figure 15 shows the output and transfer characteristics curve. The good agreement between our modeling results and the experimental data has been observed [86,87]. Figure 16 shows the drain conductance and trans-conductance curves [86,87]. Our model well agrees with the measured results.

(a)

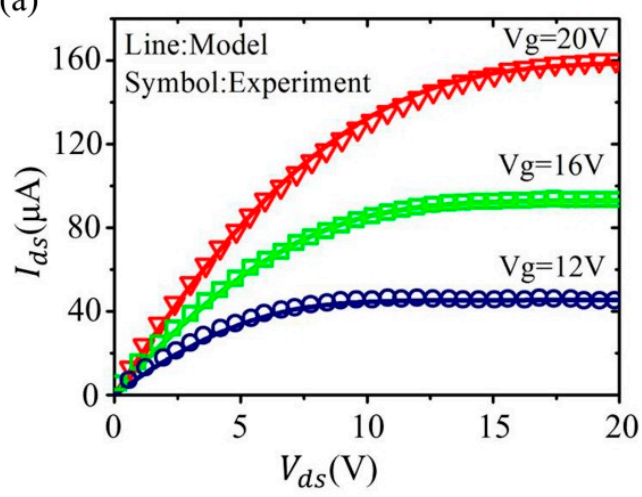

(b)

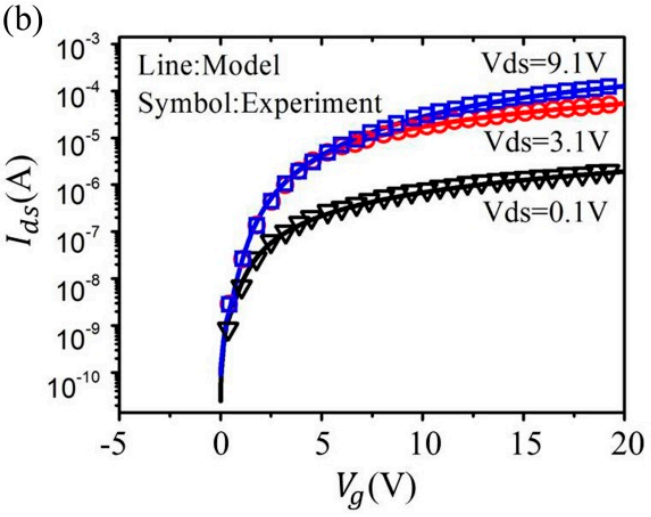

Figure 15. Comparison between the calculation and experimental data; (a) for output characteristics of In-Ga-Zn-O (IGZO) under different gate voltages; and (b) transfer characteristics of IGZO under different drain-source voltages. 
(a)

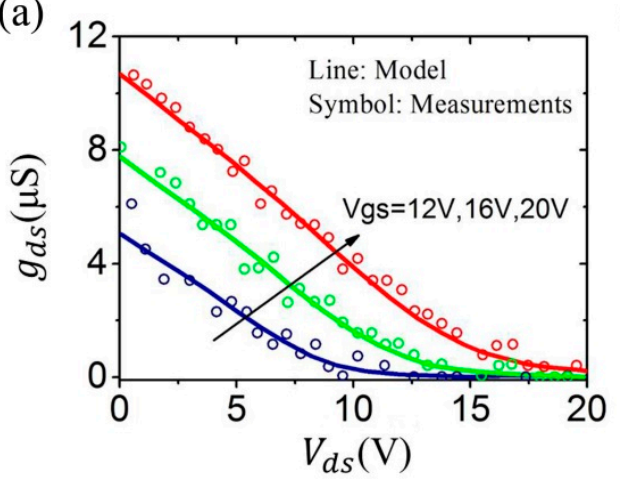

(b)

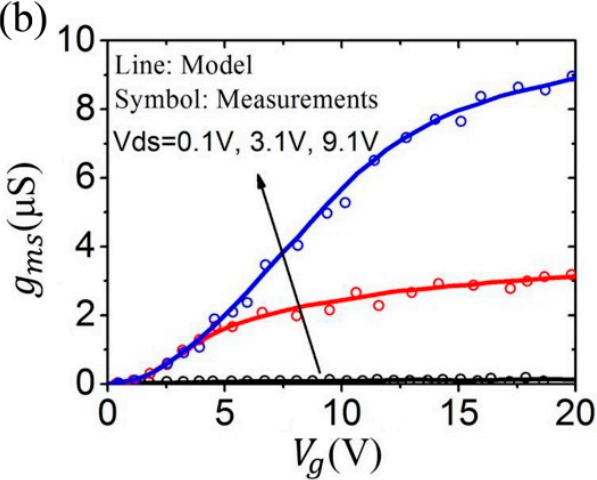

Figure 16. Model $g_{d s}-V_{d s}$ curves (a) and trans-conductance curves (b).

\section{Comparison of Various Compact Models}

As mentioned above, the most difference between silicon-based TFTs and TFTs with new active material (e.g., OTFTs and IGZO TFTs) derived from the fact that whether the threshold voltage can be defined in TFT device. Since OTFTs and IGZO TFTs usually operate in accumulation region, the formulation of compact model should discard the influence of the threshold voltage. The following will give a comparison for different compact models.

\subsection{Comparison of Model Accuracy}

For the compact models, the central aim is to accurately and physically describe the current-voltage characteristics of TFTs. Here, we will discuss various compact models and their accuracies verified for TFTs. For polysilicon TFTs, we firstly compare $V_{d s}-I_{d s}$ characteristics based on the surface-potential-based by Chen et al. [72] and the EMA method by Iñiguez et al. [28], respectively, as shown in Figure 17. It is obvious that the surface-potential-based model agrees well with experimental data. However, the simulated results from Iñiguez et al. show a well consistent between model and experiment under low drain voltage, with increasing the drain voltage, the model seriously deviated from the experiment. Similar errors of model accuracy have also been found in the OTFT compact models. Figure 18 shows a comparison of $V_{d s}-I_{d s}$ characteristics based on the surface potential and the generic model, respectively [24,83]. For the surface-potential-based model in Figure 18a, the accuracy is good in all regions, but for the generic model in Figure 18b the errors increase with the gate voltage increasing.

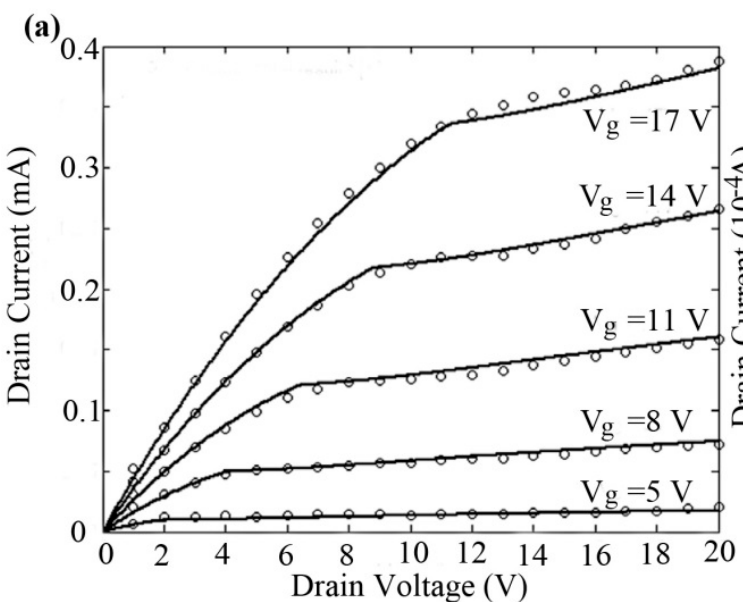

(b)

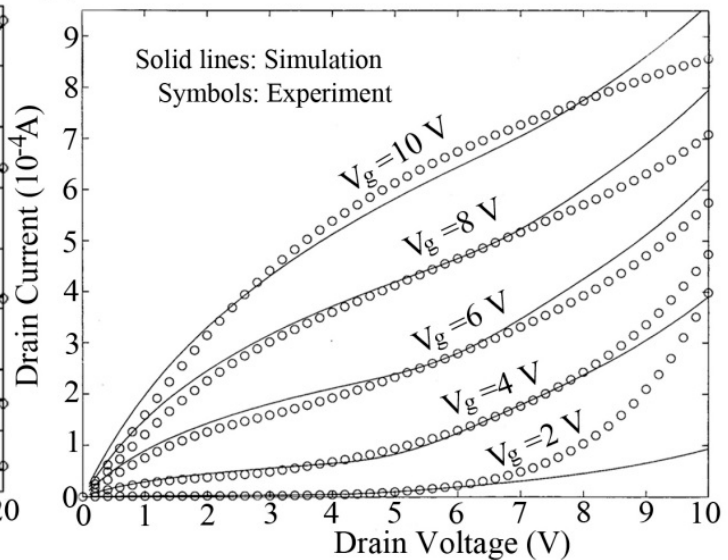

Figure 17. Comparison of $V_{d s}-I_{d s}$ of polysilicon TFTs based on the surface-potential from Chen et al. (a), and the effective medium approximation from Iñiguez et al. (b). 
(a)

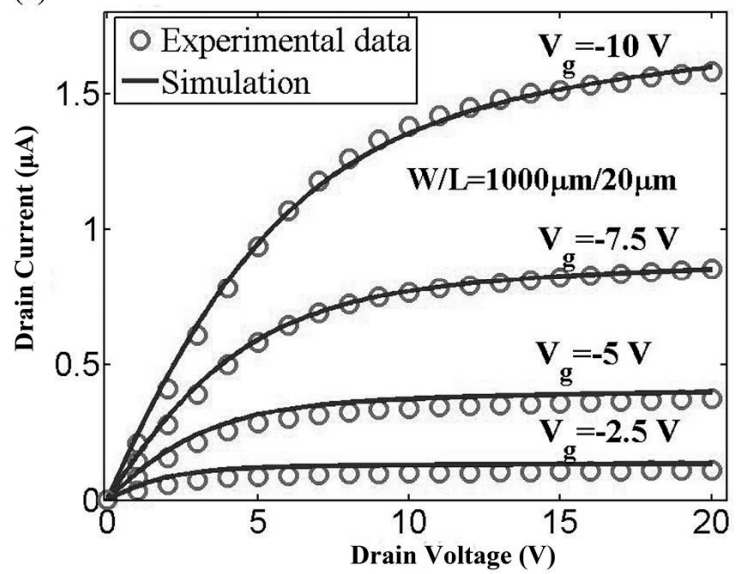

(b)

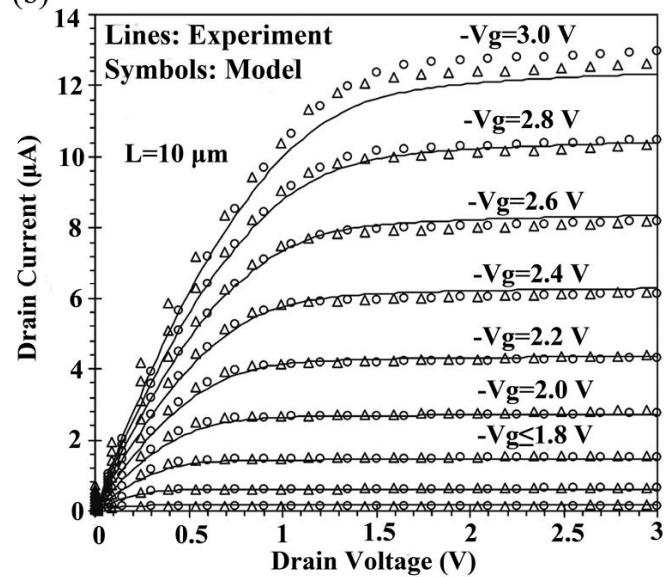

Figure 18. Comparison of $V_{d s}-I_{d s}$ of OTFTs based on the surface-potential (a), and the generic model (b), respectively.

Strictly speaking, the errors derive from the transformation from the numerical equation to the analytical solution. To obtain analytical solution, the authors usually transferred the numerical model to analytical expression by using some reasonable assumption. Intuitively, various assumptions will generate different error values, which finally affect the model accuracy. Thus, the analysis of the error is essential to transfer the numerical equation to the analytical expression. For the surface-potential-based compact models, reducing the errors of the calculated surface potential has become an important criterion. However, as compared with surface-potential-based models, the errors for the charge-based models are always ignored, which thus results in the lower accuracy.

\subsection{Parameter Comparison and Extraction}

Apart from the accuracy and comprehensive nature, an excellent compact model should include as few parameters as possible fitting the TFT characteristics. Tables 1 and 2 give a summary of the parameter comparison for the surface-potential-based compact models and OTFT compact models based on different approaches, respectively. It is found that the researchers always aspired for as few parameter numbers as possible during developing compact models. Actually, for the compact model, the fewer the non-physical parameters (fitting parameters), the better the model is considered. From Tables 1 and 2, one can see that the parameter numbers in our model is just 12, which is superior, compared with other models.

Table 1. Comparison of fitting parameter numbers for the surface-potential-based compact models.

\begin{tabular}{cccc}
\hline Types & Parameter Numbers & Authors & Years \\
\hline \multirow{2}{*}{ Polysilicon TFTs } & $15[67]$ & Y. Shimizu, et al. & 2006 \\
& $14[72]$ & R. S. Chen, et al. & 2007 \\
& $11[32]$ & W. L. Deng, et al. & 2011 \\
\hline \multirow{2}{*}{ Amorphous TFTs } & $16[80]$ & Y. Liu, et al. & 2008 \\
& $22[89]$ & Y. Liu, et al. & 2009 \\
\hline \multirow{2}{*}{ Organic TFTs } & $14[81]$ & J. Qin, et al. & 2014 \\
\hline \multirow{2}{*}{ IGZO TFTs } & $12[83]$ & Our work & 2015 \\
& $14[90]$ & A. Tsormpatzoglou, et al. & 2013 \\
\hline
\end{tabular}


Table 2. Comparison of fitting parameter numbers for OTFT compact model based different approaches.

\begin{tabular}{cccc}
\hline Years & Parameter Numbers & Authors & Method \\
\hline 1995 & $14[39]$ & M. S. Shur, et al. & Effective medium approach \\
1999 & $28[27]$ & B. Iñiguez, et al. & Effective medium approximation \\
1999 & $14[29]$ & M. D. Jacunski, et al. & Semi-empirical approach \\
2006 & $15[67]$ & Y. Shimizu, et al. & Surface potential \\
2007 & $14[72]$ & R. S. Chen, et al. & Surface potential \\
2007 & $12[30]$ & W. J. Wu, et al. & Generation-recombination model \\
2011 & $11[32]$ & W. L. Deng, et al. & Surface potential \\
2015 & $12[83]$ & Our work & Surface potential \\
\hline
\end{tabular}

In addition to using as few parameters as possible, parameter extraction also plays an important role in understanding TFT characteristics. Generally speaking, parameter extraction aims at being physical. To achieve higher level, the parameter sequence should introduce physical effect [21]. However, considering the continuity and accuracy of compact model, the fitting parameters will be used for smoothing the output curves and reducing the error. It is anticipated that the compact models of TFTs with the parameter setting will be suitable to circuit design and can provide accurate insight into the performance. The main criterion for a good set of parameters is the balance of error, efficiency and continuity. For IGZO TFTs, we have developed an extraction flow of the key physical parameters of the surface-potential-based compact model, as shown in Figure 19 [86,87]. Based on the corresponding equations shown in Figure 19, four key parameters can be extracted, that is, the maximum mobility $\mu_{0}$, the characteristic temperature $T_{T A}$, the product of the escape frequency $v_{0}$ and carrier lifetime $\tau_{0}$.

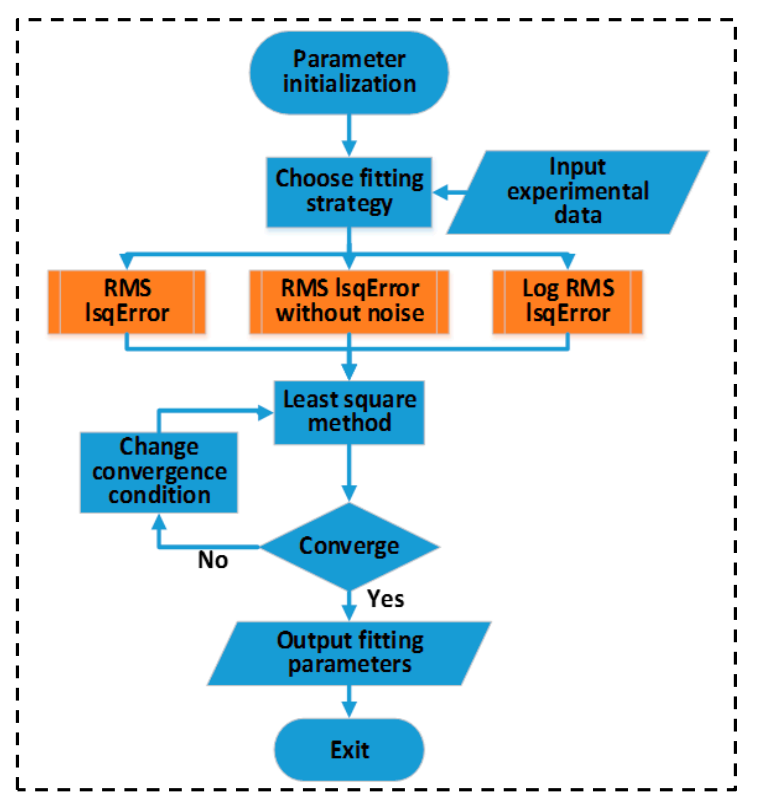

Figure 19. Extraction flow of key physical parameters of the model.

\subsection{Criterion and Continuous Test of Compact Models}

A compact model must satisfy several rather restrictive requirements imposed by their use in advanced circuit simulators. From the mathematical point of view, the equations of the models should meet three classes at least [40], that is, "class 1" in order to be compatible with Newton-Raphson-based circuit simulators, with "class 2 " or better preferred in order to achieve faster convergence, and "class 3" required for circuit simulation of active-matrix organic light-emitting diode (AMOLED) displays or distortion modeling in RF circuits. Currently, the most compact models are satisfied to the "class 1". A small numbers of compact models can meet the requirements of "class 2" and "class 3" together. For the "class 3 " requirement, the application is completely based on the active layers of TFTs. 
For example, silicon-based TFTs (poly-Si and a-Si:H) are mainly used in AMOLED displays. OTFTs can be applied to logic circuit design and flat-panel display. IGZO TFTs can be used in constructing RFID tags or inverter. Thus, the compact model of TFTs should be established according to their application.

In addition, it would be specially mentioned that, in order to meet the requirement of "class 2", the compact model must fulfill one of the benchmark tests, i.e., Gummel symmetry test (GST) [21,91,92]. Based on our surface-potential-based compact model for IGZO TFTs, the GST has been provided [86,87], as shown in Figure 20. Figure 20a shows a GST circuit for IGZO TFTs. Generally, the higher-order derivatives in TFT compact models are obtained as a function of $V_{x}$, which is symmetry for $V_{x}=0$. This symmetry roots in the symmetry device structure and channel. Figure 20b shows the GST for the 1, 2, 3-order derivative of the drain current of IGZO TFTs, which display a good continuity and symmetry. Thus, our compact model in IGZO TFTs can pass the GST.

(a)

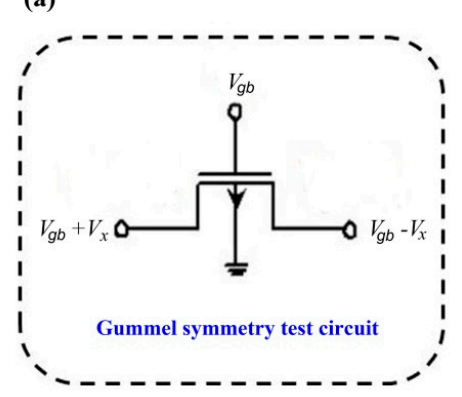

(b)

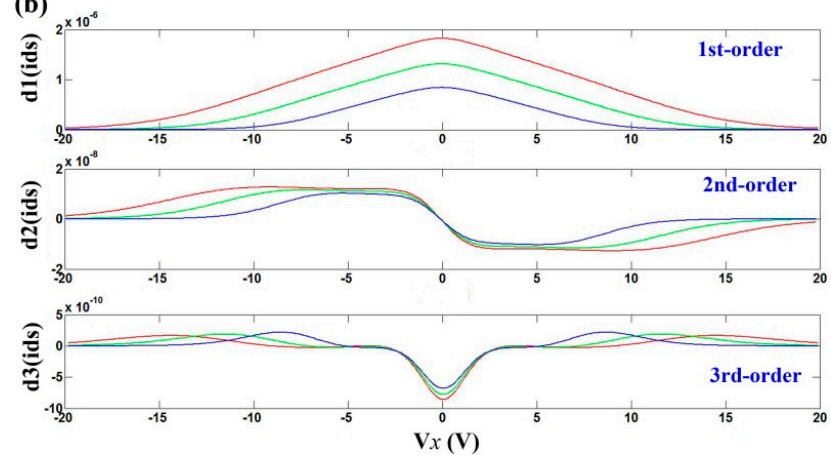

Figure 20. (a) Gummel symmetry test circuit for IGZO TFTs; and (b) Gummel symmetry test for the 1, 2, 3-order derivative of the drain current under different gate voltages.

\section{Conclusions and Outlook}

Compact models form a critical link between the manufacturing teams and the chip design teams by mathematically capturing the properties of devices. We have reviewed the concept, development and application of compact model of TFTs. Based on different active materials in TFTs, the charge transport characteristics has also been discussed in detail. Based on the different approaches, especially the surface-potential-based, the merits and shortcomings for current compact models have discussed. We also proposed our surface-potential-based compact models for organic and IGZO TFTs and parameter extraction technology. The comparison of various compact models has been summarized.

Currently, the compact model is still open and evolving. To achieve the excellent compact model, the following should be considered: accurate in all regions of operation and types, suitable for all simulation modes, excellent convergence, and intuitive and easy to extract parameters. In addition, to keep pace with the increase of circuit operating frequencies and device tolerances scale down, the compact model of TFTs should account for the bias dependent contact resistances, gate tunneling, interface effect and scaling effect. The dynamic behavior, aging and hysteresis of TFTs also should be considered in developing the compact model to pursue the future circuit design.

Author Contributions: N.L. and L.L. conceived the idea and designed this work; N.L. drafted the paper; N.L., and L.L. discussed the results and commented on the manuscript; all authors have given approval to the final version of the manuscript.

Acknowledgments: This work was supported in part by National key research and development program (Grant Nos. 2016YFA0201802, 2017YFB0701703, 2018YFA0208503), by the Opening Project of Key Laboratory of Microelectronic Devices and Integrated Technology, Institute of MicroElectronics Chinese Academy of Sciences, by the Beijing Training Project for the Leading Talents in S\&T under Grant No. Z151100000315008, and by the National Natural Science Foundation of China (Grant Nos. 61725404, 61574166, 61874134, 61221004, 61376112, and 61404164), by International cooperation project of CAS under Grant 172511KYSB20150006, and by the Strategic Priority Research Program of Chinese Academy of Sciences (Grant No. XDB30000000, XDB12030400).

Conflicts of Interest: The authors declare no conflict of interest. 


\section{References}

1. Kanatzidis, M.G. Quick-set Thin Films. Nature 2004, 428, 269-270. [CrossRef] [PubMed]

2. Lu, N.D.; Wei, W.; Chuai, X.C.; Li, L.; Liu, M. Carrier thermoelectric transport model for black phosphorus field-effect transistors. Chem. Phys. Lett. 2017, 678, 271-274. [CrossRef]

3. Wang, L.; Wang, W.; Xu, G.; Ji, Z.; Lu, N.; Li, L.; Liu, M. Analytical Carrier Density and Quantum Capacitance for Graphene. Appl. Phys. Lett. 2016, 108, 013503. [CrossRef]

4. Saremi, M. Carrier Mobility Extraction Method in ChGs in the UV Light Exposure. Micro Nano Lett. 2016, 11, 762-764. [CrossRef]

5. Saremi, M.; Rajabi, S.; Barnaby, H.J.; Kozicki, M.N. The Effects of Process Variation on the Parametric Model of the Static Impedance Behavior of Programmable Metallization Cell (PMC). MRS Proc. 2014, 1692, 9-39. [CrossRef]

6. Saremi, M. A Physical-based Simulation for the Dynamic Behavior of Photodoping Mechanism in the Chalcogenide Materials Used in the Lateral Programmable Metallization Cells. Solid State Ion. 2016, 290, 1-5. [CrossRef]

7. Horowitz, G. Organic Thin Film Transistors: From Theory to Real Devices. J. Mater. Res. 2004, 19, $1946-1962$. [CrossRef]

8. Liu, W.Y.; Wu, Q.S.; Ren, Y.K.; Cui, P.; Yao, B.B.; Li, Y.B.; Hui, M.; Jiang, T.Y.; Bai, L. On the bipolar DC flow field-effect-transistor for multifunctional sample handing in microfluidics: A theoretical analysis under the Debye-Huckel limit. Micromachines 2018, 9, 82. [CrossRef] [PubMed]

9. RSporea, A.; Trainor, M.J.; Young, N.D.; Shannon, J.M.; Silva, S.R.P. Source-gated transistors for order-of-magnitude performance improvements in thin-film digital circuits. Sci. Rep. 2014, 4, 4295. [CrossRef] [PubMed]

10. Itoh, T.; Kobayashi, A.; Ueno, K.; Ohta, J.; Fujioka, H. Fabrication of InGaN thin-film transistors using pulsed sputtering deposition. Sci. Rep. 2016, 6, 29500. [CrossRef] [PubMed]

11. Weimer, P.K. The TFT-A New Thin-Film Transistor. Proc. IRE 1962, 50, 1462-1469. [CrossRef]

12. Fortunato, E.; Barquinha, P.; Pimented, A.; Goncalves, A.; Marques, A.; Pereira, L.; Martins, R. Recent Advances in ZnO Transparent Thin Film Transistors. Thin Solid Films 2005, 487, 205-211. [CrossRef]

13. LeComber, P.G.; Spear, W.E.; Gaith, A. Amorphous-Silicon Field-Effect Device and Possible Application. Electron. Lett. 1979, 15, 179-181. [CrossRef]

14. Depp, S.W.; Juliana, A.; Huth, B.G. Polysilicon FET Devices for Large Area Input/Output Application. In Proceedings of the 1980 International Electron Devices Meeting, Washington, DC, USA, 8-10 December 1980; p. 703.

15. Tsumura, A.; Koezuka, H.; Ando, T. Macromolecular Electronic Device: Field-Effect Transistor with a Polythiophene Thin Film. Appl. Phys. Lett. 1986, 49, 1210. [CrossRef]

16. Xu, W.; Hu, Z.H.; Liu, H.M.; Lan, L.F.; Peng, J.B.; Wangn, J.; Cao, Y. Flexible all-organic, all-solution processed thin film transistor array with ultrashort channel. Sci. Rep. 2016, 6, 29055. [CrossRef] [PubMed]

17. Nomura, K.; Ohta, H.; Takagi, A.; Kamiya, T.; Hirano, M.; Hosono, H. Room-Temperature Fabrication of Transparent Flexible Thin-Film Transistors Using Amorphous Oxide Semiconductors. Nature 2004, 432, 488-492. [CrossRef] [PubMed]

18. Han, K.; Qiao, G.H.; Deng, Z.L.; Zhang, Y.N. Asymmetric drain extension dual-kk trigate underlap FinFET based on RF/Analog circuit. Micromachines 2017, 8, 330. [CrossRef] [PubMed]

19. Li, J.F.; Mao, S.M.; Xu, Y.H.; Zhao, X.D.; Wang, W.B.; Guo, F.J.; Zhang, Q.F.; Wu, Y.Q.; Zhang, B.; Chen, T.S. An Improved Large Signal Model for $0.1 \mu \mathrm{m}$ AlGaN/GaN High Electron Mobility Transistors (HEMTs) Process and Its Applications in Practical Monolithic Microwave Integrated Circuit (MMIC) Design in W band. Micromachines 2018, 9, 396. [CrossRef] [PubMed]

20. Buccella, P.; Stefanucci, C.; Zou, H.; Moursy, Y.; Iskander, R.; Sallese, J.M.; Kayal, M. Methodology for 3-D Substrate Network Extraction for SPICE Simulation of Parasitic Currents in Smart Power ICs. IEEE Trans. Comput.-Aided Des. Integr. Circuits Syst. 2016, 35, 1489-1502. [CrossRef]

21. Lu, N.D.; Wang, L.F.; Li, L.; Liu, M. A Review for Compact Model of Graphene Field-Effect Transistors. Chin. Phys. B 2017, 26, 036804. [CrossRef]

22. Krishnamoorthy, S.; Chowdhury, M.H. Investigation and a practical compact network model of thermal stress in integrated circuits. Integr. Comput.-Aided Eng. 2009, 16, 131-140. [CrossRef] 
23. Available online: https://www.src.org/program/grc/ds/research-needs/2003/compact-modeling.pdf (accessed on 14 April 2003).

24. Marinov, O.; Deen, M.J.; Zschieschang, U.; Klauk, H. Organic Thin-Film Transistors: Part I-Compact DC Modeling. IEEE Trans. Electron Dev. 2009, 56, 2952-2961. [CrossRef]

25. Kacprzak, T.; Materka, A. Compact DC Model of GaAs FETs for Llarge-Signal Computer Calculation. IEEE J. Solid-State Circuits 1983, 18, 211-213. [CrossRef]

26. Ahmed, S.; Kim, D.; Shichijo, H. A Comprehensive Analytic Model Accumulation-Mode MOSFETs in Polysilicon Thin Films. IEEE Trans. Electron Devices 1986, 33, 973-985. [CrossRef]

27. Siddiqui, M.J.; Qureshi, S. Surface-Potential-Based Charge Sheet Model for The Polysilicon Thin Film Transistors without Considering Kink Effect. Microelectron. J. 2001, 32, 235-240. [CrossRef]

28. Iñiguez, B.; Xu, A.; Fjeldly, T.A.; Shur, M.S. Unifed Model for Short-Channel Poly-Si TFTs. Solid-State Electron. 1999, 43, 1821-1831. [CrossRef]

29. Jacunski, M.D.; Shur, M.S.; Owusu, A.A. A Short-Channel DC SPICE Model for Polysilicon Thin-Film Transistors Including Temperature Effects. IEEE Trans. Electron Devices 1999, 46, 1146-1159. [CrossRef]

30. Wu, W.J.; Yao, R.H.; Li, S.H.; Hu, Y.F.; Deng, W.L.; Zheng, X.R. A Compact Model for Polysilicon TFTs Leakage Current Including the Poole-Frenkel Effect. IEEE Trans. Electron Devices 2007, 54, 2975-2984. [CrossRef]

31. Lee, M.; Tai, C.W.; Huang, J.J. Correlation between Gap State Density and Bias Stress Reliability of Nanocrystalline TFTs Comparing with Hydrogenated Amorphous Silicon TFTs. Solid-State Electron. 2013, 80, 72-75. [CrossRef]

32. Deng, W.L.; Huang, J.K. A Physics-Based Approximation for the Polysilicon Thin-Film Transistor Surface Potential. IEEE Electron. Dev. Lett. 2011, 32, 647-649. [CrossRef]

33. Li, L.; Debucquoy, M.; Genoe, J.; Heremans, P. A Compact Model for Polycrystalline Pentacene Thin-Film Transistor. J. Appl. Phys. 2010, 107, 024519. [CrossRef]

34. Hao, Z.W.S.; Duval, J.; Ravelosona, D.; Klein, J.O.; Kim, J.V.; Chappert, C. A Compact Model of Domain Wall Propagation for Logic and Memory Design. J. Appl. Phys. 2011, 109, 07D501. [CrossRef]

35. Valletta, A.; Demirkol, A.S.; Maira, G.; Frasca, M.; Vinciguerra, V.; Occhipinti, L.G.; Fortuna, L.; Mariucci, L. A Compact SPICE Model for Organic TFTs and Applications to Logic Circuit Design. IEEE Trans. Nanotech. 2016, 15, 754-761. [CrossRef]

36. Hsieh, H.H.; Kamiya, T.; Nomura, K.; Hosono, H.; Wu, C.C. Modeling of Amorphous InGaZnO4 Thin Film Transistors and Their Subgap, Density of States. Appl. Phys. Lett. 2008, 92, 133503. [CrossRef]

37. Ghittorelli1, M.; Torricelli1, F.; Steen, J.J.V.D.; Garripoli, C.; Tripathi, A.; Gelinck, G.H.; Cantatore, E.; Kovács-Vajna, Z.M. Physical-Based Analytical Model of Flexible a-IGZO TFTs Accounting for Both Charge Injection and Transport. In Proceedings of the 2015 IEEE International Electron Devices Meeting (IEDM), Washington, DC, USA, 7-9 December 2015; Volume 28, pp. 723-726.

38. Duavll, S.G. Statical Circuit Modeling and Optimization. In Proceedings of the IEEE 2000 5th International Workshop on Statistical Metrology, Honolulu, HI, USA, 10 June 2000.

39. Ortiz-Conde, A.; García-Sánchez, F.J.; Muci, J.; Malobabic, S.; Liou, J.J. A Review of Core Compact Models for Undoped Double-Gate SOI MOSFETs. IEEE Trans. Electron Devices 2007, 54, 131-140. [CrossRef]

40. Gildenblat, G.; Li, X.; Wu, W.M.; Wang, H.L.; Jha, A.; Langevelde, R.V.; Smit, G.D.J.; Scholten, A.J.; Klassen, D.B.M. PSP: An Advanced Surface-Potential-Based MOSFET Model for Circuit Simulation. IEEE Trans. Electron Devices 2006, 53, 1979-1994. [CrossRef]

41. Song, J.; Yu, B.; Yuan, Y.; Taur, Y. A Review on Compact Modeling of Multiple-Gate MOSFETS. IEEE Trans. Electron Devices 2009, 56, 1858-1869.

42. Cheng, X.; Lee, S.; Yao, G.Y.; Nathan, A. TFT Compact Modeling. J. Disp. Technol. 2016, 12, 898-906. [CrossRef]

43. Baccarani, G.; Riccò, B. Transport Properties of Polycrystalline Silicon Films. J. Appl. Phys. 1978, 49, 5565-5571. [CrossRef]

44. Seto, J.Y.W. The Electrical Properties of Polycrystalline Silicon Films. J. Appl. Phys. 1975, 46, 5247-5254. [CrossRef]

45. Rech, B.; Wagner, H. Potential of Amorphous Silicon for Solar Cells. Appl. Phys. A 1999, 69, $155-157$. [CrossRef]

46. Bagolini, L.; Mattoni, A.; Collins, R.T.; Lusk, M.T. Carrier Localization in Nanocrystalline Silicon. J. Phys. Chem. C 2014, 118, 13417-13423. [CrossRef] 
47. Lu, N.D.; Li, L.; Liu, M. Universal Carrier Thermoelectric-Transport Model Based on Percolation Theory in Organic Semiconductors. Phys. Rev. B 2015, 91, 195205. [CrossRef]

48. Li, L.; Lu, N.D.; Liu, M.; Bässler, H. General Einstein Relation Model in Disordered Organic Semiconductors under Quasiequilibrium. Phys. Rev. B 2014, 90, 214107. [CrossRef]

49. Lu, N.D.; Li, L.; Banerjee, W.; Liu, M. Physical Model of Seebeck Coefficient under Surface Dipole Effect in Organic Thin-Film Transistors. Org. Electron. 2016, 29, 27-32. [CrossRef]

50. Lu, N.D.; Li, L.; Liu, M. A Review of Carrier Thermoelectric-Transport Theory in Organic Semiconductors. Phys. Chem. Chem. Phys. 2016, 18, 19503-19525. [CrossRef] [PubMed]

51. Ambegaokar, V.; Halperin, B.I.; Langer, J.S. Hopping Conductivity in Disordered Systems. Phys. Rev. B 1971, 4, 2612-2620. [CrossRef]

52. Miller, A.; Abrahams, E. Impurity Conduction at Low Concentrations. Phys. Rev. 1960, 120, 745-755. [CrossRef]

53. Klauk, H. Organic Thin-Film Transistors. Chem. Soc. Rev. 2010, 39, 2643-2666. [CrossRef] [PubMed]

54. Nomura, K.; Kamiya, T.; Ohta, H.; Ueda, K.; Hirano, M.; Hosono, H. Carrier Transport in Transparent Oxide Semiconductor with Intrinsic Structural Randomness Probed Using Single-crystalline $\mathrm{InGaO}_{3}(\mathrm{ZnO})_{5}$ Films. Appl. Phys. Lett. 2004, 85, 1993-1995. [CrossRef]

55. Germs, W.C.; Adriaans, W.H.; Tripathi, A.K.; Roelofs, W.S.C.; Cobb, B.; Janssen, R.A.J.; Gelinck, G.H.; Kemerink, M. Charge Transport in Amorphous InGaZnO Thin-Film Transistors. Phys. Rev. B 2012, 86, 155319. [CrossRef]

56. Lu, N.; Li, L.; Sun, P.; Banerjee, W.; Liu, M. A Unified Physical Model of Seebeck Coefficient in Amorphous Oxide Semiconductor Thin-Film Transistors. J. Appl. Phys. 2014, 116, 104502. [CrossRef]

57. Li, L.; Lu, N.D.; Liu, M. Field Effect Mobility Model in Oxide Semiconductor Thin Film Transistors With Arbitrary Energy Distribution of Traps. IEEE Electron Device Lett. 2014, 35, 226-228. [CrossRef]

58. Podzorov, V. Charge Carrier Transport in Single-crystal Organic Field-Effect Transistors. In Organic Field Effect Transistor; CRC Press: Boca Raton, FL, USA, 2007; pp. 27-72.

59. Sze, S.M.; Ng, K.K. Physics of Semiconductor Devices, 3rd ed.; Wiley: New York, NY, USA, 2007.

60. Jung, L.; Damiano, J.; Zaman, J.R.; Batra, S.; Manning, M.; Banerjee, S.K. A Leakage Current Model for Sub-Micron Lightly-Doped Drain-Offset Polysilicon TFTs. Solid-State Electron. 1995, 38, 2069-2073. [CrossRef]

61. Khakzar, K.; Lueder, E.H. Modeling of Amorphous-Silicon Thin-Film Transistors for Circuit Simulations with SPICE. IEEE Trans. Electron Devices 1992, 39, 1428-1435. [CrossRef]

62. Faughnan, B. Subthreshold Model of A Polycrystalline-Silicon Thin Film Field-Effect Transistor. Appl. Phys. Lett. 1987, 50, 290-292. [CrossRef]

63. Shur, M.S.; Slade, H.C.; Jacunski, M.D.; Owusu, A.A.; Ytterdal, T. SPICE Models for Amorphous Silicon and Polysilicon Thin Film Transistors. J. Electrochem. Soc. 1997, 144, 2833-2839. [CrossRef]

64. Siddiqui, M.J.; Qureshi, S. An Empirical Model for Leakage Current in Poly-Silicon Thin Film Transistor. Solid State Electron. 2000, 44, 2015-2019. [CrossRef]

65. Iñiguez, B.; Fjeldly, T.A.; Shur, M.S. Thin Film Transistor Modeling, in Silicon and Beyond: Advanced Circuit Simulators and Device Models; Shur, M.S., Fjeldly, T.A., Eds.; World Scientific Publishers: Singapore, 2000; pp. 703-723.

66. Tsividis, Y.P.; Suyama, K. MOSFET Modeling for Analog Circuit CAD: Problems and Prospects. IEEE J. Solid-State Circuits 1994, 29, 210-216. [CrossRef]

67. Tsuji, H.; Kuzuoka, T.; Kishida, Y.; Kirihara, M.; Kamakura, Y.; Morifuji, M.; Shimizu, Y.; Miyano, S.; Taniguchi, K. A New Surface Potential Based Poly-Si TFT Model for Circuit Simulation. In Proceedings of the 2006 International Electron Devices Meeting, San Francisco, CA, USA, 11-13 December 2006; pp. 179-186.

68. Leroux, T. Static and Dynamic Analysis of Amorphous-Silicon Field-Effect Transistors. Solid-State Electron. 1986, 29, 47-58. [CrossRef]

69. Brews, J.R. A Charge-Sheet Model of the MOSFET. Solid-State Electron. 1978, 21, 345-355. [CrossRef]

70. Sleight, J.W.; Rios, R. A Continuous Compact MOSFET Model for Fully- and Partially-Depleted SOI Devices. IEEE Trans. Electron Devices 1998, 45, 821-825. [CrossRef]

71. Pao, H.C.; Sah, C.T. Effects of Diffusion Current on Characteristics of Metal-Oxide (Insulator)-Semiconductor Transistors. Solid-State Electron. 1966, 9, 927-937. [CrossRef] 
72. Chen, R.S.; Zheng, X.R.; Deng, W.L.; Wu, Z.H. A Physics-Based Analytical Solution to the Surface Potential of Polysilicon Thin Film Transistors Using the Lambert W Function. Solid-State Electron. 2007, 51, 975-981. [CrossRef]

73. Corless, R.M.; Gonnet, G.H.; Hare, D.E.G.; Jeffrey, D.J.; Knuth, D.E. On Lambert's W function. Adv. Comput. Math. 1996, 5, 329-359. [CrossRef]

74. G Gildenblat, T.L.C.; Gu, H.W.; Cai, X. SP: An Advanced Surface Potential-Based Compact MOSFET Model. IEEE J. Solid-State Circuits 2004, 39, 1394-1406. [CrossRef]

75. Hack, M.; Shaw, J. Transient Simulations of Amorphous-Silicon Devices, Materials Research Soc. Symp. Proc. 1991, 219, 315-320. [CrossRef]

76. Shur, M.S.; Jacunski, M.D.; Slade, H.C.; Hack, M. Analytical Models for Amorphous-Silicon and Polysilicon Thin-Film Transistor for High-Definition-Display Technology. J. Soc. Inf. Disp. 1995, 4, 223-236. [CrossRef]

77. Servati, P.; Nathan, A. Modeling of the Static and Dynamic Behavior of Hydrogenated Amorphous Silicon Thin-Film Transistors. J. Vac. Sci. Technol. A 2002, 20, 1038-1042. [CrossRef]

78. Colalongo, L. A New Analytical Model for Amorphous-Silicon Thin-Film Transistors Including Tail and Deep States. Solid-State Electron. 2001, 45, 1525-1530. [CrossRef]

79. Servati, P.; Striakhilev, D.; Nathan, A. Above-Threshold Parameter Extraction and Modeling for Amorphous Silicon Thin-Film Transistors. IEEE Trans. Electron Devices 2003, 50, 2227-2235. [CrossRef]

80. Liu, Y.; Yao, R.H.; Li, B.; Deng, W.L. An Analytical Model Based on Surface Potential for a-Si:H Thin-Film Transistors. J. Disp. Technol. 2008, 4, 180-187.

81. Qin, J.; Yao, R.H. A Physics-Based Scheme for Potentials of a-Si:H TFT with Symmetric Dual Gate Considering Deep Gaussian DOS Distribution. Solid-State Electron. 2014, 95, 46-51. [CrossRef]

82. Bässler, H. Charge Transport in Disordered Organic Photoconductors, a Monte Carlo Simulation Study. Phys. Status Solidi B 1993, 175, 15-56. [CrossRef]

83. Wang, L.; Lu, N.D.; Li, L.; Ji, Z.Y.; Banerjee, W.; Liu, M. Compact Model for Organic Thin-Film Transistor with Gaussian Density of States. AIP Adv. 2015, 5, 047123. [CrossRef]

84. Coehoorn, R.; Pasveer, W.F.; Bobbert, P.A.; Michels, M.A.J. Charge-Carrier Concentration Dependence of The Hopping Mobility in Organic Materials with Gaussian Disorder. Phys. Rev. B 2005, 72, 155206. [CrossRef]

85. Li, L.; Chung, K.-S.; Jang, J. Field Effect Mobility Model in Organic Thin Film Transistor. Appl. Phys. Lett. 2011, 98, 023305. [CrossRef]

86. Zong, W.W.; Li, L.; Jang, J.; Lu, N.D.; Liu, M. Analytical Surface-Potential Compact Model for Amorphous-IGZO Thin-Film Transistors. J. Appl. Phys. 2015, 117, 215705. [CrossRef]

87. Zong, Z.W.; Li, L.; Jang, J.; Li, Z.G.; Lu, N.D.; Shang, L.W.; Ji, Z.Y.; Liu, M. A New Surface Potential-Based Compact Model for a-IGZO TFTs in RFID Applications. In Proceedings of the 2014 IEEE International Electron Devices Meeting, San Francisco, CA, USA, 15-17 December 2014; Volume 35, pp. 860-863.

88. Ghittorelli, M.; Torricelli, F.; Colalongo, L.; Vajna, Z.M.K. Accurate analytical physical modeling of amorphous InGaZnO thin-film transistors accounting for trapped and free charges. IEEE Trans. Electron Devices 2014, 61, 4105-4112. [CrossRef]

89. Liu, Y.; Yao, R.H.; Li, B.; Xie, W.N. A Physical Model Based on Surface Potential for Double-Gate a-Si:H TFTs. In Proceedings of the 2009 IEEE International Conference of Electron Devices and Solid-State Circuits (EDSSC), Xi'an, China, 25-27 December 2009.

90. Tsormpatzoglou, A.; Hastas, N.A.; Choi, N.; Mahmoudabadi, F.; Hatalis, M.K.; Dimitriadis, C.A. Analytical Surface-Potential-Based Drain Current Model for Amorphous InGaZnO Thin Film Transistors. J. Appl. Phys. 2013, 114, 184502. [CrossRef]

91. Bendix, P.; Rakers, P.; Wagh, P.; Lemaitre, L.; Grabinski, W.; McAndrew, C.; Gu, X.; Gildenblat, G. RF distortion analysis with compact MOSFET models. In Proceedings of the IEEE 2004 Custom Integrated Circuits Conference (IEEE Cat. No.04CH37571), Orlando, FL, USA, 6 October 2004; pp. 9-12.

92. McAndrew, C.C. Validation of MOSFET Model Source-Drain Symmetry. IEEE Trans. Electron Devices 2006, 53, 2202-2206. [CrossRef]

(C) 2018 by the authors. Licensee MDPI, Basel, Switzerland. This article is an open access article distributed under the terms and conditions of the Creative Commons Attribution (CC BY) license (http:/ / creativecommons.org/licenses/by/4.0/). 